This item was submitted to Loughborough's Research Repository by the author.

Items in Figshare are protected by copyright, with all rights reserved, unless otherwise indicated.

\title{
Enhancing polymer electrolyte membrane fuel cell system diagnostics through semantic modelling
}

PLEASE CITE THE PUBLISHED VERSION

https://doi.org/10.1016/j.eswa.2020.113550

PUBLISHER

Elsevier

VERSION

AM (Accepted Manuscript)

PUBLISHER STATEMENT

This paper was accepted for publication in the journal Expert Systems with Applications and the definitive published version is available at https://doi.org/10.1016/j.eswa.2020.113550

LICENCE

CC BY-NC-ND 4.0

\section{REPOSITORY RECORD}

Tsalapati, Eleni, C Johnson, Tom Jackson, Lisa Jackson, Derek Low, Ben Davies, Lei Mao, and Andrew West. 2020. "Enhancing Polymer Electrolyte Membrane Fuel Cell System Diagnostics Through Semantic Modelling”. Loughborough University. https://hdl.handle.net/2134/12408644.v1. 


\title{
Enhancing Polymer Electrolyte Membrane Fuel Cell System Diagnostics through Semantic Modelling
}

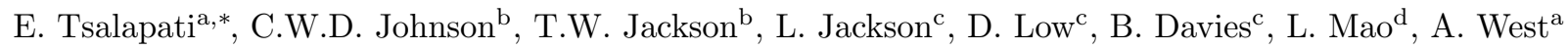 \\ ${ }^{a}$ School of Mechanical, Electrical and Manufacturing Engineering Loughborough University, Epinal Way, Loughborough, \\ LE11 3TU, UK. \\ ${ }^{b}$ School of Business and Economics, Loughborough University, Epinal Way, Loughborough, LE11 3TU, UK. \\ ${ }^{c}$ School of Aeronautical and Automotive Engineering, Loughborough University, Epinal Way, Loughborough, LE11 $3 T U$, UK. \\ ${ }^{d}$ School of Engineering Science, University of Science and Technology of China, Hefei 230027, China
}

\begin{abstract}
Polymer electrolyte membrane fuel cells (PEMFC) are a promising technology for economic and environmentally friendly energy production. However, they haven't reached their full potential in the market yet as only few reliable PEMFC systems have successfully passed the prototyping face. A drawback of the current diagnostic tools is that only a select few are of high genericity, reliability and can perform efficiently on-line at the same time. Furthermore, there is only limited research identifying both PEMFC stack faults and ancillary system faults simultaneously. While none of the existing tools can be interrogated by the end-user. In this research, we develop novel artificial intelligence-based technologies to overcome these existing barriers, i.e., i) a semantically enriched integrating schema (ontology) of the overall operation and structure of the PEMFC that allows automatic inference engines to automatically deduce fault detection; ii) a knowledgebased, light-weight, on-line fuel cell system diagnosis (FuCSyDi) platform. FuCSyDi detects and provides the location of failures by considering only the data from the reliable sensors. Additionally, it provides the reasons underpinning any forthcoming failures and enables the end-user to interrogate the platform for further information regarding its operation and structure. Our platform is validated by performing tests against common automotive stress conditions. This innovative approach enhances the reliability of the fuel cell system diagnosis and, hence, its lifetime performance.
\end{abstract}

Keywords: PEMFC, Semantic technologies, System monitoring, Ontology Based Data Access, Diagnostic system

\section{Introduction}

Fuel cells are a zero-emission, efficient and high-quality energy source that provide a future economically competitive option with respect to conventional energy sources. Their silent, efficient and clean operation makes them great alternative power sources for a wide variety of applications; from light-duty vehicles and heavy-duty trucks to hospitals and power stations. According to the US Department of Energy (DOE), in 2017 fuel cell shipments reached half a gigawatt and 62,000 fuel cell systems were shipped worldwide, while at the same time, the emerging fuel cell industry topped 1,6 billion in USD in just one year. Established fuel cell technologies include the alkaline fuel cell, solid oxide fuel cell, phosphoric acid fuel cell and the Polymer Electrolyte Membrane fuel cell (PEMFC). This research paper is focused on PEMFC, as they dominate the fuel cell shipments almost by a quarter ${ }^{1}$.

\footnotetext{
${ }^{*}$ Corresponding author.

Email addresses: E.Tsalapati@lboro.ac.uk (E. Tsalapati), c.w.d.johnson@lboro.ac.uk (C.W.D. Johnson), t.w.jackson@lboro.ac.uk (T.W. Jackson), l.m.jackson@lboro.ac.uk (L. Jackson), d.low@lboro.ac.uk (D. Low), b.davies2@lboro.ac.uk (B. Davies), leimao82@ustc.edu.cn (L. Mao), a.a.west@lboro.ac.uk (A. West)

${ }^{1}$ https://www .californiahydrogen.org/wp-content/uploads/2019/01/TheFuelCellIndustryReview2018.pdf
} 
Although the PEMFC technology is among the most promising cell types, especially in the transportation industry $^{2}$, the PEMFC market has not reached its full potential yet. One reason for this is that only a few reliable fuel cell systems have successfully passed the prototyping face and hence brought to the market (Wang, 2017). Even so, extensive studies on the durability of the PEMFC systems' components have shown that some of them tend to degrade faster than expected (Wang, 2017). The failure of one component can cause multiple failures to the rest of the fuel cell stack, leading to a decrease in efficiency and significantly increasing maintenance and repair costs. One way to minimise the loss of system gross performance and the cost of repair is to notify the user as soon as possible about any abnormal behaviour of the system and to provide the respective information explaining the causes of this behaviour.

In the last few years a wide range of technologies and methods have been developed in the field of health monitoring and diagnosis of PEMFCs, which mirror development in other areas such as intelligent diagnosis in machines and bearings (Zhao et al., 2019; Yang et al., 2019a). The methods can be broadly classified into three areas data-driven, model-based (white-, black- or grey- box) and knowledge-based. A drawback of both data-driven and (black-box) model-based approaches is that they are limited by the volume of the datasets including the targeted fault conditions. The generation process of these data sets can be very time consuming, especially in the case of multi-fault diagnosis, and may damage the PEMFC system (Zheng et al., 2014; Petrone et al., 2013). Additionally, the black-box model-based approaches may lack genericity (Petrone et al., 2013). White box physical models can be difficult to implement on-line due to their high level of complexity (Petrone et al., 2013). Hybrid approaches (grey-box) have received the most attention in recent years initially focusing on the use of neural networks (Asensio et al., 2017) with extension to other AI based methods (e.g. Zhao et al. (2019)).The limitation with these AI methods is the high degree of training from the data needed to ensure accurate diagnosis, acerbated with multiple failure modes.

Current knowledge-based diagnostic tools for PEMFC system diagnosis are limited to representing only the rules for fault diagnosis in a machine readable form lacking, however, any semantic content and leaving the task of interpretation and evaluation of their output to the end-user. This way, for instance, it is hard for these tools to automatically identify the location of the failure on the PEMFC system or to discern between a faulty stack and a faulty sensor. With the current fault diagnostic techniques, PEMFC faults, including PEMFC stack faults and faults of ancillary systems (e.g. sensor faults), can be identified and isolated (Zheng et al., 2013; Petrone et al., 2013; Lin et al., 2019). However, there is only limited study identifying both PEMFC stack faults and ancillary system faults simultaneously (Mao et al., 2018). The aim of this research is to fuse semantic technologies with knowledge-based methods to provide a holistic approach to health monitoring and diagnosis for PEMFC systems to enhance their capability and to maximise their lifetime potential. The semantic technologies are employed to allow seamless integration of the structural and operating data of the PEMFC system and its ancillary systems (e.g. sensors) along with the formal representation of expert's knowledge in a human and machine processible form. This way, artificialintelligence-based techniques (logical reasoning) can be applied to extract useful implied information with regards to the health state of the system enabling robust early diagnosis and decision support.

One of the most prominent semantic-web-based techniques to data access is Ontology Based Data Access (OBDA). OBDA enables the user to access and query the data, without having any a prior knowledge of the structure of the source data. This is achieved by mediating between the user and the data a formal conceptual schema, the ontology, which describes the domain of interest and constitutes the expert's knowledge. Roughly, ontologies are composed of entities and relationships between them. Their formal semantics are defined by using some knowledge representation language (e.g. Description Logics) and they ensure a precise specification of the meaning of the described elements that is both machine and human readable. The ontology along with the set of data constitute the knowledge base (KB). This way, the OBDA systems can automatically make inferences, i.e. extract implicit information, from unorganized and disconnected data with the use of powerful ontology reasoners (e.g.(Stoilos \& Stamou, 2014; Glimm et al., 2014)). Additionally, the dynamic nature of ontologies makes them easily maintainable and extendable, as long as the new knowledge does not conflict with the existing one. As a result, the model evolves with the growth of

${ }^{2}$ https://www.energy.gov/sites/prod/files/2015/11/f27/fcto_fuel_cells_fact_sheet.pdf 
knowledge without impacting dependent processes and systems. Up to today, numerous OBDA-based tools for health monitoring and diagnosis have appeared in the literature for a wide range of applications; in heavy industry (eg. petroleum, energy companies) (Kharlamov et al., 2015, 2017) to traffic congestions (Lécué et al., 2012, 2014) and expense management systems (Lécué \& Wu, 2017). However, to the best of our knowledge there is no such diagnostic system for fuel cells.

In this research paper, the novel Fuel Cell System Monitoring (FCSM) ontology is proposed. In FCSM ontology, information surrounding the structure of a fuel cell system, the specifications, the properties and the position of the components of the system, the rules for fault diagnosis as presented by Davies et al. (2017) for PEMFCs are represented formally. For the formal representation the lightweight Description Logic based language OWL 2 EL is used. On top of the FCSM ontology the OBDA-based Fuel Cell System Diagnosis platform (FuCSyDi) is proposed, which is a health monitoring and diagnostic tool for PEMFC systems. In brief, FuCSyDi displays on-line the health state of an PEMFC system, detects the unreliable sensors, identifies any abnormality of the system, by taking under consideration only the measurements of the reliable sensors and alerts the user about any forthcoming failures presenting at the same time the abnormalities and the location in the system that they occur. Additionally, it serves as an interface through which the user can interrogate the KB to obtain further knowledge concerning the functionality and the structure of the fuel cell system and its monitoring sensors. It is interesting to note that the limited number of sensors monitoring a fuel cell system allows us to introduce a software system simple in structure, i.e. lightweight, thus highly efficient. To keep FuCSyDi lightweight, only real-time data and a restricted part of the historical data are exploited.

This research extends the work conducted by Tsalapati et al. (2018) in the following manner: 1. The PEMFC Monitoring ontology is: a) mapped to the "Sensor, Observation, Sample, and Actuator" (SOSA) ontology (Atkinson et al., 2017), which is endorsed by W3C, to establish a shareable and semantically strong ontology; b) extended to capture more details on the functionality and the specifications of the PEMFC stack and of the related sensors; c) extended to incorporate the full set of failure modes studied by Davies et al. (2017), besides the water management issues; d) improved to enhance the automation of the diagnostic process; and 2. The FuCSyDi platform is: a) extended to allow the user to perform queries; b) extended to perform sensor reliability checking before alerting the user; c) tested with a new set of data. The key innovations of the method over those that currently exist are its flexibility and adaptability with easy extension of the rules to facilitate new developments in technology or failure mode understanding, coupled with advanced information retrieval and inferencing capabilities enabling more detailed diagnosis. These attributes combined with the on-line diagnosis functionality enable prompter and the most appropriate mitigation strategies to be implemented, which is especially important for faults where degradation effects are reversible.

This paper is structured as follows. First, to emphasize the novelty of this work an overview of the related work is presented in Section 2. Then, after a brief introduction on the semantic technologies and on fuel cells in Section 3, the description of the FCSM ontology follows in Section 4. In Section 5 the full functionality of the fuel cell prognosis and diagnosis platform FuCSyDi is presented. Finally, the evaluation of the system is in Section 6 and the conclusions and future work are outlined in Section 7.

\section{Related Work}

Within the research efforts for the development of fuel cells in recent years, an increase of the application of intelligent control strategies has been seen, including the use of advanced diagnostic tools. Diagnostic techniques are generally classified as either (a) data-driven, that use either data-mining or statistical methods, (b) model-based, which create some model which is used for evaluation, or (c) knowledge-based, which calls upon a heuristic understanding of the system functionality.

Data-driven approaches use historical observations of performance to make diagnostic assessments by using some data processing method. There are three main types of data-driven methods: the artificialintelligence based, the statistical and the signal processing-based. The goal of the artificial intelligence-based is to recognise automatically fault patterns without any prior knowledge of the system's structure (Zheng et al., 2013). They may exploit artificial neural networks (ANN) technologies, e.g. Shao et al. (2014), 
fuzzy logic, e.g. Zheng et al. (2014), or neural-fuzzy methods, e.g. (Silva et al., 2014). A drawback of the ANN and the neural-fuzzy methods is that they require large datasets under normal and faulty operating conditions. The generation of these datasets can be a very time consuming process, especially in the case of multi-faults, and can cause irreversible damage to the system (Zheng et al., 2013). Also, an existing concern (Eslamloueyan, 2011) is that the learning process of the full set of fault patterns may affect negatively the performance of such systems. The statistical methods are mostly exploited for finding the correlations among the input data and to reduce their size. For the identification of linear correlations usually the principle component analysis, e.g. (Shams et al., 2011), or the Fischer discriminant analysis, e.g. (He et al., 2005), are used. For non-linear correlations the Kernel-methods are preferred, which however are very time consuming (Zheng et al., 2013). Bayesian networks are traditional data processing probabilistic tools that are used as statistical classifiers in diagnostic of fuel cell, e.g. (Wasterlain et al., 2010). Although accurate, they require a large amount of input data (Lin et al., 2019). Other classification tools used for PEMFC diagnosis include the support vector machines (SVM), e.g. (Li et al., 2016). Finally, the signal processing methods extract features from the sensor data, and identify unique fuel cell operational states, e.g. (Mao et al., 2017). A common drawback of the data-based approaches is that they can only diagnose the previously experienced faults.

The aim of the model-based approaches is to develop a system model which can be used to calculate the residuals between the system in operation and a theoretical system in healthy mode. Three main types of models appear in the literature: the analytical models (white-box), the models that are derived only from experimental practice (black-box) and the grey-box which are a combination of white- and black-box approaches. The white-box methods, e.g. (Damour et al., 2015), employ complex mathematical equations to simulate the systems behaviour. Recent approach use advanced multi-domain bond graph models to consider the complexities of fuel cell operation, and provide the diagnostic signatures for each failure mode (Jha et al., 2016; Vasilyev et al., 2015). Models of overall fuel cell system performance have also been provided with Petri-nets using Monte Carlo simulations (Wieland et al., 2009; Whiteley et al., 2015; Fecarotti et al., 2016). Due to their high mathematical complexity, analytical models are usually preferred for off-line tasks like system understanding, off-line monitoring and training simulators (Petrone et al., 2013). In black-box models the correlation between the inputs and the outputs is derived from suitable experimental databases. They may use techniques based on ANN, e.g. (Pourkiaei et al., 2016), neuro-fuzzy,e.g. (Rahimzadeh et al., 2016) or SVM (Kheirandish et al., 2016). Use of AI-based methods have now exploded with use of methods like artificial neural networks both feed-forward and recurrent (Jeppesen et al., 2017; Zhao et al., 2019; Ma et al., 2018), extreme and deep learning methods (Yang et al., 2019b; Liu et al., 2019), clustering methods including support vector and k-means (Liu et al., 2018). The black-box methods are better suited for complex non-linear systems, but they require a large amount of experimental data (Petrone et al., 2013) and only the SVM-based ones are characterised by a high level of genericity. The grey-box models are based on the analytical models but by replacing some of the complex mathematical equations with couplings between parameters, e.g. (Andreasen et al., 2011). Although, the grey-box models demonstrate accurate results, have higher genericity that most of the black-box approaches, and can perform efficiently on-line, their development is still at early stage (Petrone et al., 2013). A general drawback of most of the above methods is that they lacks easy interpretability for the user.

Knowledge-based methods are based on expert knowledge gathered from the scientific literature and the experimental experience. Luta \& Raji (2019) used fuzzy inference system techniques to compare the time response of various types of maximum power point tracking controllers. Zheng et al. (2014) performed the diagnosis classifications by using fuzzy logic and a rule base of expert knowledge. Davies et al. (2017) developed a diagnostic system based on fuzzy logic to cover the main degradation phenomena appearing in PEMFC usage. However, similarly to the aforementioned studies, it did not take under consideration the potential damage of the sensors and neither provided to the user the causes of the failure of the system. Kamal \& Aitouche (2018) propose a sensor fault estimation method for PEMFC using TS fuzzy model with disturbance and faults. However, the fuel cell model is developed using mathematical equations, introducing similar difficulties to the white-box models.

This research seeks to contribute in this area by adopting an innovative knowledge-based approach, employing logical reasoning to identify failures, as well as assessing the degradation of the fuel cell performance 
at system and component levels. In particular, it takes the research of Davies et al. (2017) one step further, integrating the heuristic knowledge that is available in the literature with logical reasoning to attempt to enhance fuel cell reliability by taking into account only the reliable sensors. The use of semantics allows greater interoperability in the diagnostic reasoning for the operator and user in a real-time diagnostic output. Specifically, not only it provides the user with a holistic overview of the system's (and not only of the stack) functionality and alerts about any forthcoming degradation state along with the respective explanations, but also it allows the operator to interrogate the knowledge base for further information.

\section{Preliminaries}

For better understanding of the rest of this research paper, in this section we introduce all necessary notions related to semantic web technology and to PEMFCs. For further details on semantic web technology the reader can refer to Antoniou et al. (2012).

\subsection{Semantic Web Technology}

Terminology of Ontologies. An ontology is a conceptual schema that describes formally the domain of interest. The vocabulary of the ontology is defined by the (atomic) classes, the individuals and the properties. The classes represent abstract groups, sets, or collections of objects, the individuals refer to the real-world concrete objects, and the properties relate objects either to objects (object properties) or to data values (data properties).

Example 3.1. Let $\mathcal{O}$ be an ontology in the domain of monitored systems. Then, the class System is used to group the various individuals that refer to systems, e.g., $\mathrm{FCS}_{01}, \mathrm{FCS}_{02}$ etc (where $\mathrm{FCS}_{X}$ symbolizes a fuel cell system with identification number $X)$, and the class Sensor to group the various sensors, e.g., sensor $_{t_{1}}$, sensor $t_{2}$ etc. Additionally, to express formally that a sensor monitors a system, the object property "monitors" is defined, with domain the class Sensor and range the class System. While, with the data property hasId the various systems and sensors are attributed with an identification code of string value, e.g. TempS,D18.

To describe further the domain of interest, the relationships between the vocabulary terms need to be defined. These relationships are called axioms. Ontologies can be regarded as sets of axioms. There are various ways of expressing formally an axiom, in this work the most user-friendly syntax is adopted, the Manchester syntax (Horridge \& Patel-Schneider, 2008), which uses the taxonomic constructor SubClassOf. For instance, the axiom Sensor SubClassOf System indicates that every individual that belongs to the Sensor class belongs also to the System class. It is important to note that a subclass always inherits the properties of its superclasses. Additionally, by using different types of constructors (e.g. the constructors or, and, only, some, value) one can define more complex axioms.

Example 3.2. Suppose that the ontology $\mathcal{O}$ described in Example 3.1 is extended by the class SensorOutput and the data properties isValid and isReliable. The domain of the is Valid property is the SensorOutput class and domain of the isReliable property is the Sensor class. The range of both properties is the set $\{$ true, false $\}$. Then, the axiom:

\section{SensorOutput and isOutputOf some(Sensor and isReliable value true)}

\section{SubClass Of isValid value true}

states that every output of a reliable sensor is valid.

Finally, an ontology can contain ontological facts or assertions. In Example 3.1, the fact that FCS $_{01}$ is of type System is described formally as System $\left(\mathrm{FCS}_{01}\right)$. Also, if we assume that the sensor $t_{1}$ monitors $\mathrm{FCS}_{01}$, then this can be expressed by the assertion monitors $\left(\right.$ sensor $_{t_{1}}, \mathrm{FCS}_{01}$ ). Similarly, the identification number of the sensor $t_{t_{1}}$ is denoted with the assertion hasId( sensor $_{t_{1}}$, TempS,D18). 
Depending on the problem, different kinds of ontologies can be developed. Upper-level (or foundation) ontologies consist of general concepts that are common across different domains. Domain ontologies are ontologies describing a specific domain of interest and can be mapped to the respective upper-level ontologies to facilitate the semantic interoperability among them.

One of the most prominent ontological languages is the Web Ontology Language OWL 2. Depending on the complexity of the class expressions appearing in the axioms, different profiles of the OWL 2 language are defined. Each profile has different expressiveness and enjoys different computational properties. In general, the more expressive a language is, the higher the complexity of the basic reasoning problems (query answering, entailment, subsumption, etc) (Baader et al., (2003). In this paper, to ensure the efficiency of the FuCSyDi platform the lightweight (PTime-complete (Baader et al., 2005)) OWL 2 EL profile (Hitzler et al., 2009 ) is used. However, one of the limitations of the OWL languages is that they do not support relations among individuals of triangle type. Hence, the information that 'if the output of a sensor monitoring a stack explains some degradation mode then the stack experiences this degradation mode' cannot be captured by OWL. To overcome this the Semantic Web Rule Language (SWRL) (Horrocks et al., 2004) is also used. SWRL rules have the form of an implication between and antecedent and a consequent. The aforementioned information can be expressed formally in SWRL as follows:

$$
\begin{array}{r}
\operatorname{Sensor}(x) \text { and Stack }(y) \text { and monitors }(x, y) \text { and hasOutput }(x, z) \text { and explains }(z, m) \\
\rightarrow \operatorname{experiences}(y, m)
\end{array}
$$

Ontology Based Data Access (OBDA). The main idea underpinning OBDA is to provide the user with access to the data sources via the use of a high-level schema, i.e. an ontology, in a terminology familiar to the user. This way, the user can formulate queries using the well-known terms defined in the ontology, without the intervention of an IT expert. An important feature of the OBDA technology is that, in contrast to the database constraints, it enriches the answers with implied information via logical reasoning over the ontology. For instance, in the fuel cell diagnosis context, given a set of diagnostic rules and an alerting mechanism for their violation, the OBDA system can answer to queries related to the cause or the causes of the deterioration or the breakdown of the system. Instead, with a database system a user, expert in both databases and fuel cells, would need to trace manually these causes.

Most of the OBDA systems can support conjunctive queries $(C Q)$. A CQ is an expression of the form

$$
Q\left(x_{1}, \ldots, x_{k}\right) \leftarrow R_{1}\left(\vec{y}_{1}\right), \ldots, R_{n}\left(\vec{y}_{n}\right)
$$

where $n \geq 0, R_{i}$ belongs to the vocabulary of the ontology, for every $i=1, \ldots, n$ and $Q$ does not appear in the ontology. The expressions $x_{1}, \ldots, x_{k}, \vec{y}_{1}, \ldots, \vec{y}_{n}$ are either variables or individuals. Also, every variable in $\left\{x_{1}, \ldots, x_{k}\right\}$ must appear in $\vec{y}_{1}, \ldots, \vec{y}_{n}$ at least once. Intuitively, expression (1) intuitively, requests for the set of individuals $x_{1}, \ldots, x_{k}$ that belong to the conjunction of the terms $R_{1}\left(\vec{y}_{1}\right), \ldots, R_{n}\left(\vec{y}_{n}\right)$. For instance, the query:

$$
Q(y, z) \leftarrow \text { FuelCellStack }(x), \text { hasId }(x, y), \text { hasTemperature }(x, z), \text { is AttachedTo }(x, u), \text { FuelCellStack }(u)
$$

requests for the identification number and the temperature of the fuel cell stacks that are attached to other fuel cell stacks.

An OBDA system translates the queries and the ontology into the vocabulary of the data sources and then performs the actual query evaluation to a suitable query answering system. In this research, we use the query answering system Hydrowl, which is one of the most efficient OBDA systems (Stoilos \& Stamou, 2014) and is partly based on GraphDB formerly known as OWLim (Bishop et al., 2011). OWLim performs the materialization technique, which given the database and the ontology, it first computes all the implied assertions that can be inferred and then returns the answers to the query.

\subsection{The PEMFC}

The polymer electrolyte membrane fuel cell (PEMFC) is an electrochemical energy conversion device. Its function is centred around the polymer electrolyte membrane, the namesake of this technology, and converts 

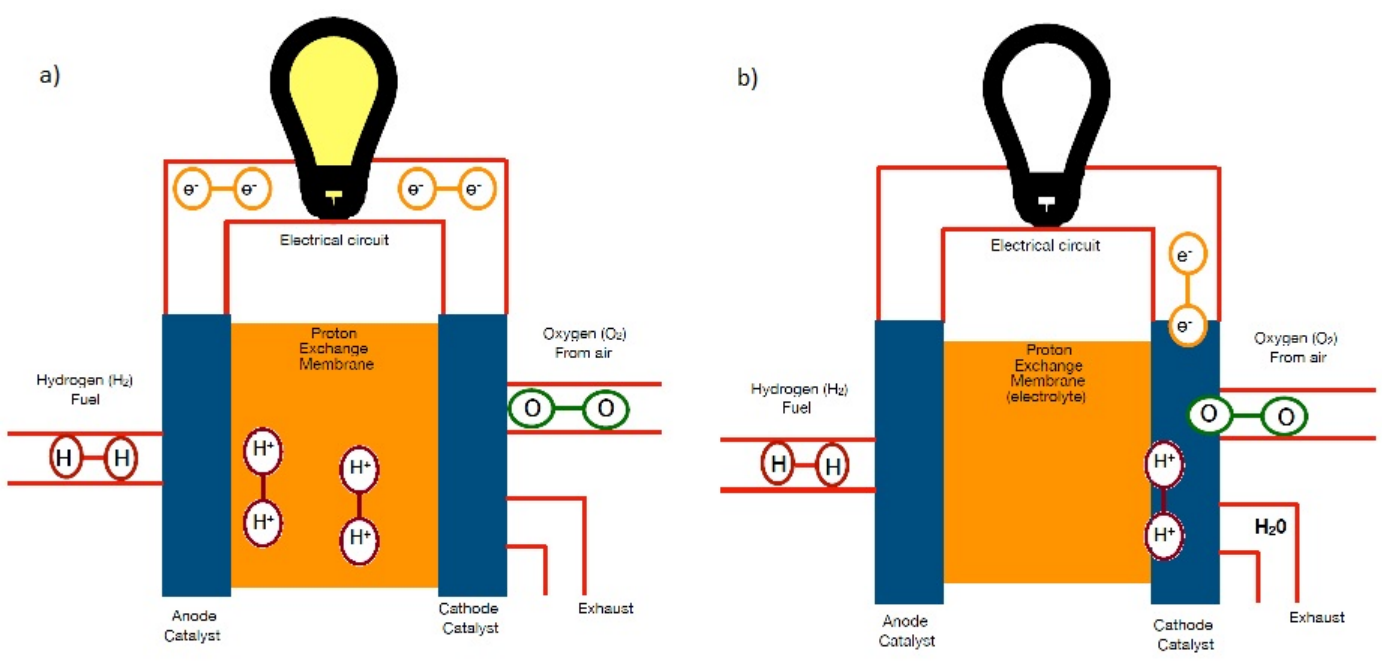

Figure 1: (a) Anode reaction: hydrogen oxidisation; (b) Cathode reaction: oxygen reduction.

chemical energy to electrical energy. A PEMFC consists primarily of three components: two electrodes, the anode and the cathode, and a conductive electrolyte, which is the polymer electrolyte membrane (PEM) and serves as ionic conductor. Each electrode is comprised of a porous, high-surface area material, usually a mixture of carbon-supported platinum and a carbon-based backing layer, coated with a catalyst layer, typically platinum or a platinum alloy. The two electrodes are insulated from each-other by the PEM.

The anode accepts the hydrogen fuel and is responsible for the hydrogen oxidation reaction. In particular, the platinum catalyst splits the hydrogen into positive ions (protons) and negatively charged electrons. The PEM allows the passage of the protons through it, from the anode to the cathode, but, since it is not electrically conductive, forces the electrons to be conducted through the catalyst layer and gas diffusion electrodes to the external circuit and eventually to the cathode creating an electrical current (Figure 1(a)). The cathode is supplied with oxygen (usually from the air) and is responsible for the oxygen reduction reaction (Larminie et al., 2003), i.e. the hydrogen ions and the electrons combine with the oxygen to form water, which flows out of the cell (Figure 1(b)). This is the only exhaust product from the PEMFC reaction - along with some heat - which affords the fuel cell its zero-emission reputation. The output voltage impedance presents a highly nonlinear dependence to operating conditions such as temperature, electric current, partial pressures and humidity levels of the incoming gases.

As the performance of most fuel cells is rather limited (they produce less than $0.75 \mathrm{~V}$ of electricity), to enhance the generated power, multiple cells are assembled into a fuel cell stack. The power generated by a fuel cell stack depends on the number and size of the fuel cells that comprise the stack and the surface area of the PEM. The assembled stack that contains the PEM, the catalyst layers and the gas diffusion layers constitutes the membrane electrode assembly (MEA). The degradation of these components leads to reliability problems, performance losses, and limited lifetime of the PEMFC. A small amount of degradation may be expected during normal fuel cell operation. However, off-design conditions can accelerate the impact of the degradation modes. Davies et al. (2017) have outlined a set of diagnostic rules which connect the PEMFC failure mechanisms with their accelerating operational conditions. As this set of rules constitutes a core component of the FuCSyDi tool, they are illustrated in Table 1.

The rules are based upon expert knowledge and understanding, which has been gathered directly from the literature and experimental experience. Each rule is defined in an IF-THEN form, where the IF-part of the rule expresses an abnormal operating condition (e.g. high voltage, low temperature, etc) and the THENpart of the rule the level ("evidenced"-50\%; "certain"-more than 90\%) of an occurring degradation mode. From the considered parameters, the stack voltage, stack temperature and feed humidity are monitored by the sensors. The cathode humidity, stoichiometry and humidity change are calculated from the sensor 
Table 1: Diagnostic Rules for PEMFC (Davies et al., 2017)

\begin{tabular}{|c|c|c|}
\hline Rule & IF & THEN \\
\hline 1 & Stack voltage is low & Flooding is evidenced \\
\hline 2 & Stack temperature is cold & Flooding is certain \\
\hline 3 & Cathode humidity is high & Flooding is certain \\
\hline 4 & Cathode humidity is low & Dehydration is certain \\
\hline 5 & Stack temperature is hot & Dehydration is certain \\
\hline 6 & Anode stoichiometry is low & Carbon support corrosion is evidenced \\
\hline 7 & Anode humidity change is large & Membrane mechanical stress is evidenced \\
\hline 8 & Cathode humidity change is large & Membrane mechanical stress is evidenced \\
\hline 9 & $\begin{array}{l}\text { Anode humidity change is small AND } \\
\text { Cathode humidity change is small }\end{array}$ & Membrane mechanical stress is evidenced \\
\hline 10 & $\begin{array}{l}\text { Anode humidity change is large AND } \\
\text { Cathode humidity change is large }\end{array}$ & Membrane mechanical stress is certain \\
\hline 11 & Stack voltage is high & Catalyst dissolution is evidenced \\
\hline 12 & $\begin{array}{c}\text { Stack voltage is very high AND } \\
\text { Stack voltage cycle number is low }\end{array}$ & Catalyst dissolution is evidenced \\
\hline 13 & $\begin{array}{l}\text { Stack voltage is very high AND } \\
\text { Stack voltage cycle number is high }\end{array}$ & Catalyst dissolution is certain \\
\hline 14 & Stack voltage is high & Membrane chemical breakdown is evidenced \\
\hline 15 & Stack voltage is very high & Membrane chemical breakdown is certain \\
\hline
\end{tabular}




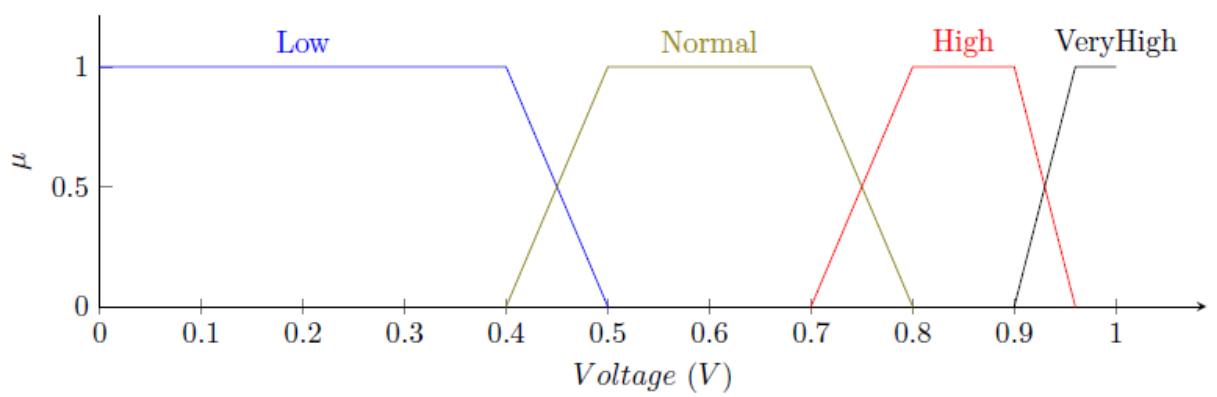

Figure 2: The plot for membership functions for the stack voltage (Davies et al., 2017)

Table 2: Classification thresholds for sensor outputs and calculated values for a single fuel cell

\begin{tabular}{|c|c|c|c|c|}
\hline Scale Range & Low & Normal & High & Very High \\
\hline \hline Voltage $(V)$ & $x \leq 0.45$ & $0.45<x \leq 0.75$ & $0.75<x \leq 0.93$ & $x>0.93$ \\
\hline CathodeHumidity $(\%)$ & $x \leq 17$ & $17<x \leq 100$ & $100<x$ & - \\
\hline AnodeHumidityChange & $x \leq 2.5$ & $2.5<x \leq 5.5$ & $5.5<x$ & - \\
\hline CathodeHumidityChange & $x \leq 2.5$ & $2.5<x \leq 5.5$ & $5.5<x$ & - \\
\hline AnodeStoichiometry & $x \leq 0.5$ & $0.5<x \leq 3.25$ & $3.25<x$ & - \\
\hline Temperature $\left({ }^{\circ} \mathrm{C}\right)$ & $x \leq 20$ & $20<x \leq 90$ & $90<x$ & - \\
\hline
\end{tabular}

outputs, according to the fuel cell formulary presented by Kabza (2016). In particular, the calculation of the cathode humidity is based on the relative humidity and the temperature of the air in the cathode. Stoichiometry is calculated using the ratio between reactant feed and consumption, based upon current production. Humidity change is the ratio of water molecules to membrane active sites, and its calculation is based on the relative humidity level.

The range of values that classify the operational conditions to a scale of low-normal-high-very high are established from Davies et al. (2017) by extracting the common factors from knowledge in the literature and in practice. In particular, Davies et al. have defined the fuzzy set boundaries for each linguistic term by using trapezoidal membership functions. For instance, the membership function for the stack voltage, which is presented in Figure 2, is derived from the definitions of "low" $(<0.4 \mathrm{~V})$ (Ramaswamy et al., 2008; Chung et al., 2009a), "normal" (0.5-0.7 V) (Ramos-Paja et al., 2009; Ramaswamy et al., 2008; Borup et al., 2007; Khan \& Iqbal, 2005; Chung et al., 2009b), and "very high" (> 0.95 V) (Ferreira et al., 2005; Inaba et al., 2006; Sompalli et al., 2007) voltage range found in the literature; the "high" range lies between the "normal" and the "very high".

The classification presented in Table 2 is obtained by converting the fuzzy membership functions to crisp values when a stack contains a single PEMFC. This conversion is achieved by defining the crisp boundaries from the intersections of the corresponding membership functions, as they are plotted by Davies et al. in such a way that at the intersection point each fuzzy variable has 0.5 degree of membership. For instance, in Figure 2, at the intersection of "Low" and "Normal" membership functions, which corresponds to $0.45 \mathrm{~V}$, both "Low" and "Normal" have 0.5 degree of membership, i.e. the $0.45 \mathrm{~V}$ is regarded as $50 \%$ "Low" and $50 \%$ "Normal". Hence, without loss of generality, is this research work voltages $\leq 0.45 \mathrm{~V}$ are regarded as low and $>0.45 \mathrm{~V}$ as normal.

From the parameters appearing in Table 2, only the voltage depends on the number of fuel cells contained in the stack; for a fuel cell stack with $n$ fuel cells, the voltage thresholds are multiplied by $n$. The rest of the parameters remain invariant. It is also important to note that the thresholds presented in Table 2 are for one voltage cycle number (where the cycle number is increased as soon as the voltage passes the threshold of $0.95 n \mathrm{~V}$, for $n$ fuel cells).

The first five rules of Table 1 detail problems that may arise from water management strategies. As the polymer membrane requires a water content in order to achieve proton-conductivity, reactant gasses 
are often humidified, to maintain hydration within the cell. However, there is a balance to be achieved as condensation and evaporation of water are quick degradation phenomena. The first rule indicates that high current loading generates water as a reaction product while the next four rules consider the flooding and dehydration phenomena through temperature and humidity changes. The next rule shows that, under fuel starvation conditions, the carbon-structure can be corrupted. The next four rules reflect the fact that water content can induce fatigue stress across the area of the membrane that ultimately leads to pinholes. The four rules that follow, consider the platinum nano-particle catalyst in the PEMFC. The nano-particles agglomerate together to reduce high surface energy, however the rate is significantly increased when the voltage is high or when it is driven in a cyclical profile (stack voltage cycles are counted when the operating voltage passes $0.9 \mathrm{~V}$ ). Finally, the last two rules relate to open circuit operation, which is known to generate hydroxyl radicals which is regarded as responsible for highly reactive attacks to the membrane polymer chain. These rules are based on the latest understanding of fuel cell failure modes and additional rules can be derived with the inclusion of additional sensors and the enhanced clarity of multiple failure mode effects.

\section{The Fuel Cell System Monitoring Ontology}

The purpose of this section is to define the semantic layer through which the end-user will have two possible interactions: a) access to the knowledge implied from the raw data, and b) a reliable diagnostic mechanism enriched with semantic context. The raw data includes the streaming data from the sensors, the static data from the technical descriptions and the specifications of the monitored systems, and a short amount (predefined by the user) of historic data for the reliability testing of the sensors.

As one of the key factors that determine the efficiency of the reasoning processes is the expressivity of the ontology, there is a clear trade-off between the level of detail of the captured knowledge and the efficiency of the platform. Hence, to produce a highly efficient prognostic and diagnostic system, the aim is to develop an ontology that will capture all the necessary knowledge related to system monitoring in the simplest possible way. In particular, the ontology must aggregate all the information surrounding: a) the parts of the specification and the functionality of the fuel cell system that are of interest to the end-user; and b) the diagnostic rules presented by Davies et al. (2017).

One of the most widely used (Roda \& Musulin, 2014; Kharlamov et al., 2014) upper ontologies for the semantic representation of sensors and the information surrounding them is the Semantic Sensor Network (SSN) ontology (Compton et al., 2012), which is developed by the W3C Semantics Sensor Networks Incubator Group. Unfortunately though, its high expressiveness (2NExpTime-complete) makes it impractical to use for the lightweight platform that is proposed in this research. The same group has developed the more lightweight "Sensor, Observation, Sample, and Actuator" (SOSA) ontology (Atkinson et al., 2017), which, however, is too simple to capture the knowledge surrounding the fuel cell system monitoring and diagnosis. Hence, we have developed the Fuel Cell System Monitoring (FCSM) ontology, which reuses some of the main concepts appearing in SOSA ontology but it is enriched with more expressivity (OWL 2 EL). In all, the FCSM ontology contains 81 classes, 33 properties and 281 logical axioms. Next, the core elements of the FCSM ontology are described in a top-down manner, i.e. starting from the most generic classes.

The graphical representation of the main classes and properties of the ontology is presented in three figures. The classes and properties surrounding the three core elements of the FCSM ontology, i.e. the classes System, State and Sensor, are depicted in Figure 3. The data properties of the main classes are depicted in Figure 4 and the part of the ontology surrounding the fuel cell system diagnosis is depicted in Figure 5. In all schemas the arrows with solid lines represent the SubClassOf relationships and the dashed arrows the properties. Initially, the elements of Figure 3 are described, starting from the class System. As in SSN and SOSA ontologies, the class System constitutes a core class of the ontology. It is a subclass of the class sosa : System and thus, is used to express pieces of infrastructure that implement a procedure (Compton et al., 2012). Such systems can be the actuators, i.e. devices that implement a procedure that changes the state of the world, and the sensors, i.e. devices that monitor the actuators and their components and return an output result. Hence, immediate subclasses of the class System are the Actuator and the Sensor class; which are subclasses of the classes sosa : Actuator and sosa : Sensor, respectively. Also, the various parts of the 


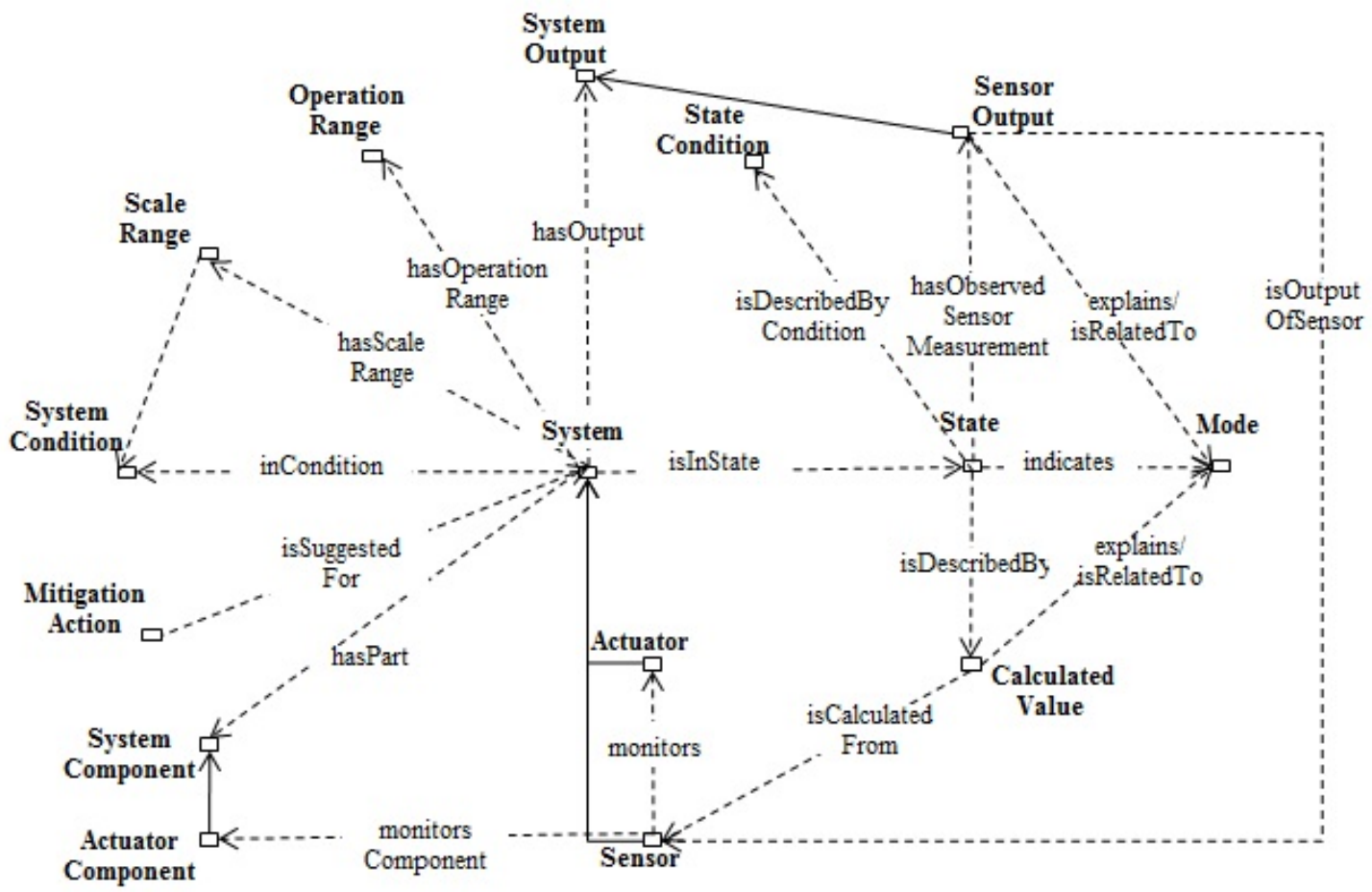

Figure 3: The graph surrounding the class System of the Fuel Cell System Monitoring Ontology

monitored system (or the actuators) are grouped with the class SystemComponent (and ActuatorComponent, respectively).

Each system is a assumed to be dynamic and, as such, can be described by a set of states which are expressed by the class State. Thus, a system ${ }_{i}$ at time $t_{j}$ is in state state@system ${ }_{i} @ t_{j}$, which is an individual of the class State. Each system has a set of output values which are captured by the class SystemOutput and the properties hasValue, hasUnit, atTime (Fig. 4); for instance, if at $t_{i}$ the output value of a system is $x$ which is measured in unit $y$ then the $x y @ t_{i}$ is an individual of the class SystemOutput and participates in the assertions hasValue $\left(x y @ t_{i}, x\right)$, hasUnit $\left(x y @ t_{i}, y\right)$ and atTime $\left(x y @ t_{i}, t_{i}\right)$. These values must be within some maximum and minimum values that indicate the healthy operation of the system. These thresholds are specific for each system and are stored in the OperationRange class by using the properties hasUpperValue, hasLowerValue (Fig. 4). For instance, the output values of a relative humidity sensor, e.g. Sensor RH, are expected to be between 0 and 100, thus if its output values violate any of these thresholds for some prespecified amount of time then the Sensor ${ }_{\mathrm{RH}}$ will be regarded as flawed (isReliable(Sensor ${ }_{\mathrm{RH}}$, false $_{\text {) }}$ ). It is important to note that a sensor may be unreliable even though its output values are within these thresholds. For instance, as it is indicated by Mao \& Jackson (2016), sudden sensor output variations (even within their normal operating range) can indicate an abnormal behaviour of the sensor when these variations do not also comply with the rest of the sensor outputs. If a sensor is unreliable then its outputs are considered as invalid, while if it is reliable its outputs are considered as valid. To express this formally the SystemOutput is also equipped with the data property isValid and the following two axioms are defined:

$$
\begin{aligned}
& \text { isOutputOf Sensor some (isReliable value false) SubClassOf isValid value false } \\
& \text { isOutputOf Sensor some (isReliable value true) SubClassOf } i s \text { Valid value true }
\end{aligned}
$$

Additionally, following Davies et al. (2017) approach, to aid the diagnostic process the sensor output values are classified to low-medium-high-very high. The defined condition indices for each sensor, against which either the measured data or the processed ones are compared, are stored by using the class ScaleRange and 

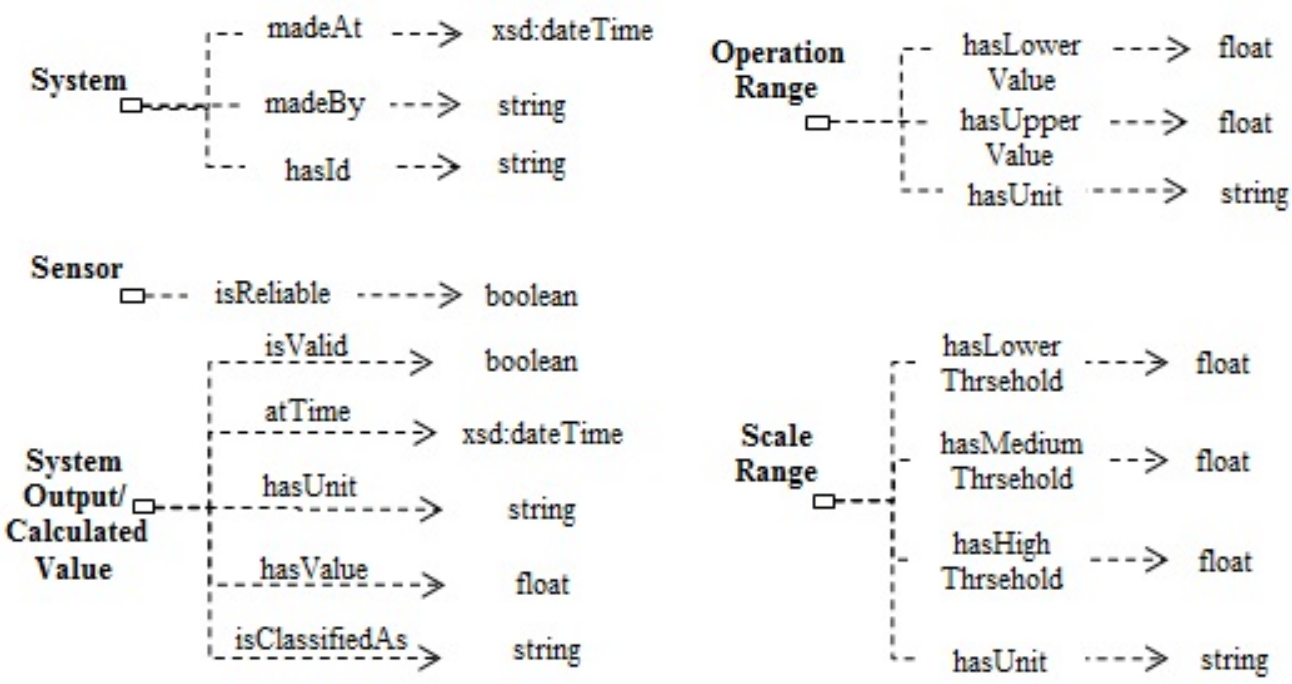

Figure 4: The main data properties of the Fuel Cell System Monitoring Ontology

the properties hasLowThreshold, hasMediumThreshold, hasHighThreshold (Fig. 4). Also, the classification type for each value is stored via the data property isClassifiedAs of the classes SystemOutput and CalculatedValueOutput.

Example 4.1. Let $\mathrm{FCS}_{01}$ be a single-cell stack which is monitored by the voltage sensor Sensor volt $_{\text {. Then, }}$ this information along with the scale ranges presented in Table 2 will be stored in the OBDA system as follows:

$$
\begin{aligned}
& \text { monitors }\left(\text { Sensor }_{\text {volt }}, \mathrm{FCS}_{01}\right) \quad \text { mediumThreshold }\left(\text { scRange }_{\text {volt }}, 0.75\right) \\
& \text { hasScaleRange(Sensor } \left.\left.\text { volt }_{\text {, }} \text { scRange }_{\text {volt }}\right) \text { highThreshold( } \text { scRange }_{\text {volt }}, 0.95\right) \\
& \text { lowThreshold (scRange } \text { volt }, 0.45) \quad \text { hasUnit }\left(\text { scRange }_{\text {volt }}, V\right)
\end{aligned}
$$

Having this information stored it is easily deduced from the first rule of Table 1 that if the voltage output is $0.35 \mathrm{~V}$, which according to the above is regarded as low, then some early traces of flooding will be detected in the stack.

However, it is important to note that the condition indices of the sensors may vary depending on the conditions under which the fuel cell system operates. For instance, the low outside temperature can reduce the overall cell voltage, thus the voltage indices may also need to be reduced. Thus, for accurate prognosis and diagnosis these conditions are captured with the two classes SystemCondition and StateCondition (both subclasses of sosa : Condition). The class SystemCondition is used for the conditions that are permanent to the system, e.g. the controlled low temperature at which a fuel cell system constantly operates, while the class StateCondition is used for the temporal conditions of the system, such as the cycle number of the stack

Finally, the class MitigationAction represents the collection of actions suggested to the end-user to prevent the overall degradation of the fuel cell system. For instance, if there is excess water in the stack and depending on its overall performance, a recommended mitigating action can be one of the following: purgeWater, blowAir, externalHeating, internalHeating. Next, the properties and the classes directly related to the class State appearing in Figure 3 are described.

Each state of the system is characterized either by the observed data from the sensors or by the processed values retrieved from the measured data. The measured data are stored in the class SensorOutput, which is a subclass of the SystemOutput. The processed values are stored in the class CalculatedValue, which enjoys the same data properties with the SystemOutput class. Similarly to the SystemOutput, the instances of the CalculatedValue class are subject to validity. In particular, if the input values required for their calculations 


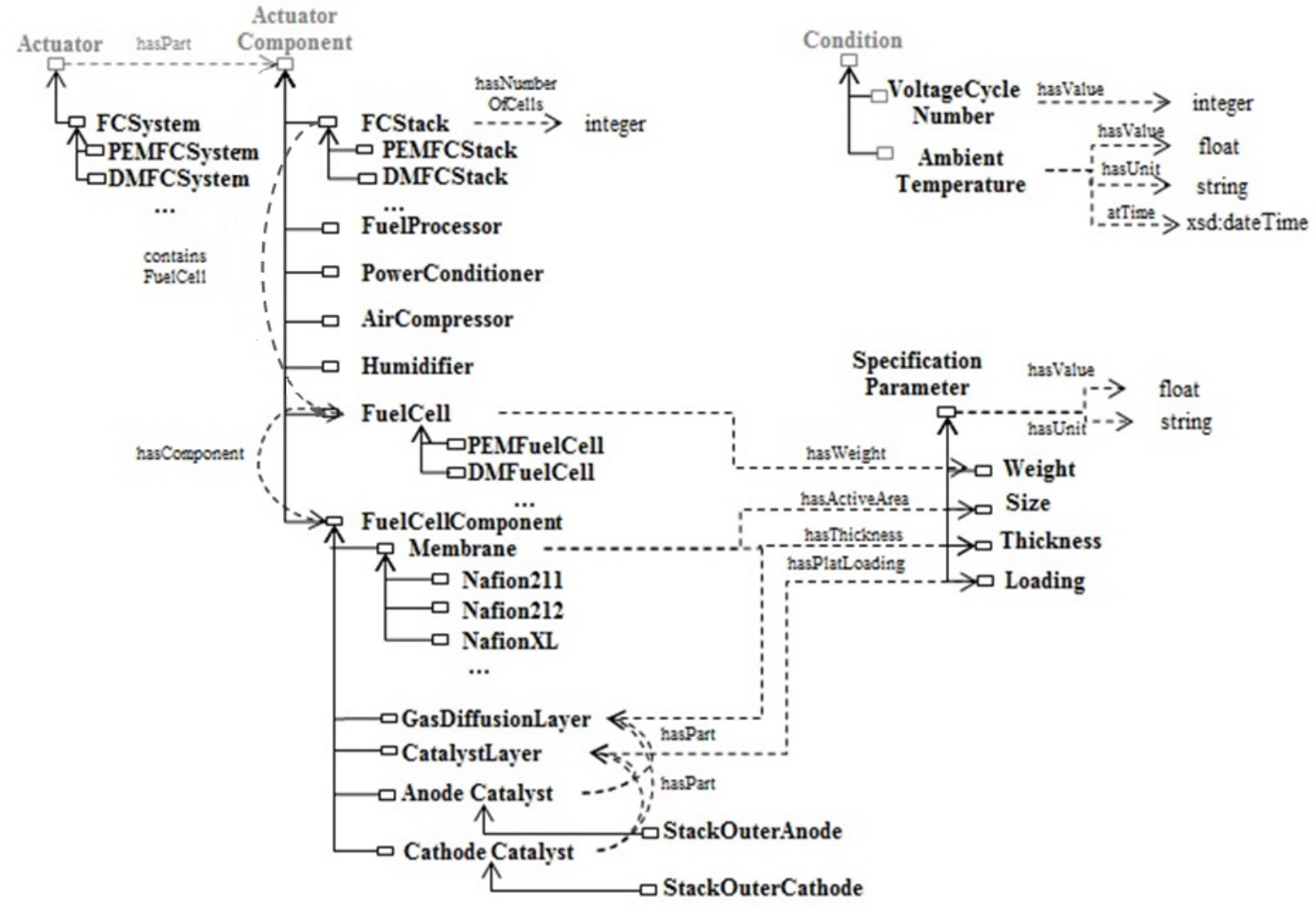

Figure 5: The graph surrounding the class FuelCellSystem of the Fuel Cell System Monitoring Ontology 
originate from unreliable sensors, then they will be regarded as invalid. Finally, each state indicates the particular Mode that a system is in at a specific time. As a particular output of a sensor (of a calculated value) can be the explanation of the degradation of the system, the sensor outputs and the calculated values are directly connected to the Mode class via the property explains. Also, there are cases were a specific abnormal value or a condition may contribute to the overall degradation of the system but does not constitute the only reason for this degradation; for these cases the property isRelatedTo is used (for visual clarity of the graph in Figure 3 the classes SystemCondition and StateCondition are not connected to the Mode class via the $i s R e l a t e d T o$ property). The various degradation modes incorporated in this ontology are the ones studied by Davies et al. (2017). Examples of the individuals of the Mode class are: healthyMode, flooding, dehydration and membraneCorrosion.

The main elements surrounding the fuel cell system diagnosis is presented in Figure 5. With a grey colour are presented the classes that appear in Figure 3 and have already been described, while the new classes are denoted with black colour. The class FCSystem represents the class of the fuel cell systems and it is a superclass of the different kinds of fuel cell systems; PEMFC systems (PEMFCSystem), direct methanol fuel cell systems (DMFCSystem), etc. Besides the stacks, there is a wide variety of additional components that accompany a fuel cell system (e.g. FuelProcessor, PowerConditioner, etc). A limited subset of these components is presented in Figure 5, however this list can be extended to contain more elements. To provide the user with an aggregated view of the PEMFC system, the ontology is enriched with its components and its specification details. The heart of a PEMFC is the membrane electrode assembly, which includes the membrane, the gas diffusion layer and the catalyst layer. Several types of membrane (Nafion 211, Nafion 212, Nafion XL, etc) are also included in the ontology. Two parts of a PEMFC stack that, according to Davies et al. (2017) are of most interest for the fuel cell system diagnosis is the anode and the cathode of the outer cells (outer anode and outer cathode, respectively), which are represented in the ontology by the terms StackOuterAnode, StackOuterCathode. The specification details include the weight of each cell, the thickness of the membranes, their active area and the platinum loading of the catalyst. Additionally, the ontology contains a set of axioms detailing the structure of the system, such as 'a fuel cell stack (FCStack) must contain at least one fuel cell'. However, for brevity their description is omitted.

Next, a core part of the ontology for the diagnostic process, which, however, is not illustrated in Figure 3 for visual clarity, is discussed. Regarding the monitoring part of the diagnostic system, there is a wide range of sensors suggested in the literature for monitoring a fuel cell system. This ontology includes only the sensors required for the diagnosis of the failure modes studied by Davies et al. (2017), however it could be expanded to incorporate more sensors. In particular, subclasses of the Sensor class are the RelativeHumiditySensor, CurrentSensor, HydrogenMassSensor, TemperatureSensor and Voltage Sensor. Hence, only the respective outputs (e.g. RelativeHumiditySensorOutput) and operating ranges are included in the ontology. Additionally, from the outputs of these sensors the stoichiometry, the cathode humidity and the humidity change (of both the anode and the cathode) are calculated. Thus, the classes StoichiometryValue, CathodeHumidityValue, HumidityChangeValue are added in the ontology as subclasses of the CalculatedValue class.

In order to take into account only the calculated values computed from the outputs of reliable sensors a set of axioms of the following form is included in the ontology:

\section{StochiometryValue and (isCalculatedFrom some (CurrentSensorand(isReliable value true))) and (isCalculatedFrom some (HydrogenMassSensorand (monitors some StacksOuterAnode) and} (isReliable value true))) SubClassOf isValid value true

Axiom (4) states that if the stoichiometry is calculated from a reliable current sensor and from a reliable hydrogen mass sensor, placed at the outer anode of the stack, then this value is valid. The axioms for the relative humidity of the anode and the cathode are constructed in a similar way.

To aid the diagnostic process all monitored operating conditions (sensor outputs and calculated values) are classified to low-medium-high (and very high when applicable) based on the respective thresholds stored in the ScaleRange class. For instance, the class TemperatureSensorOutput is a superclass of the classes: LowTemperatureSensorOutput, NormalTemperatureSensorOutput, HighTemperature-SensorOutput and 
the temperature values are classified accordingly based on the thresholds of the temperatureScaleRange, which is an instance of the ScaleRange class.

The OWL-axioms obtained from the conversion of the set of degradation rules of Table 1 to OWL are illustrated in Table 3. The first axiom states that if the voltage sensor has low output, and this measurement is valid (which, according to axiom (3), is true if the respective sensor is reliable), then this explains the evidenced flooding mode that the system experiences. The interpretation of the axioms (2)-(8), (11), (14), (15) appearing in Table 3 is similar. In axioms (9), (10), (12), (13) the respective failure mode occurs only if two different operating conditions happen at the same time. Hence, they are related to the particular state that the system is in. For instance, with the ninth axiom it is expressed that if there is a state where the stack suffers from low humidity change both at the anode and the cathode then the stack will suffer from some membrane mechanical stress. Also, in this case, each abnormal operating value contributes to the overall degradation of the system but does not constitute the only reason for this degradation; hence it is related to ev.MembraneMechanicalStress. This is expressed with the following to axioms:

\section{LowAnodeHumidityChangeValue SubClassOf isRelatedTo some ev. MembraneMechanicalStress LowCathodeHumidityChangeValue SubClassOf isRelatedTo some ev.MembraneMechanicalStress}

At the same time, it clear that if an abnormal value or condition is related to a degradation mode and describes (or is observed measurement of) a state that indicates that mode, then this value constitutes one of the explanations for this mode. This is expressed with following SWRL rule:

$$
\text { indicates }(x, y) \text { and isDescribedByCalculatedValue }(x, w) \text { and isRelatedTo }(w, y) \rightarrow \text { explains }(w, y)
$$

The structure of the respective rules for the properties hasObservedSensorMeasurement and isDescribedByCondition is similar. In the same manner, if an abnormal value or condition describes a state and can explain a specific degradation mode then the particular state indicates that mode:

$$
\begin{aligned}
& \text { State }(x) \text { and isDescribedByCalculatedValue }(x, w) \text { and explains }(w, y) \rightarrow \text { indicates }(x, y) \\
& \text { State }(x) \text { and hasObservedSensor Measurement }(x, w) \text { and explains }(w, y) \rightarrow \text { indicates }(x, y)
\end{aligned}
$$

The FCSM ontology is designed in such a way that a domain expert can further expand it depending on the nature of the fuel cell system monitored. Hence, new rules for PEMFC fault diagnosis can be added for a more robust PEMFC diagnostic system (if they are consistent with the knowledge already represented in the ontology), or it can be extended to support the diagnosis of different types of fuel cell systems as well.

Finally, the integrating nature of ontologies allows users to draw several conclusions about the operating systems, besides their condition. For instance, if the Sensor class is also enriched with information related to the manufacturing details of the sensors, i.e. manufacturing date, manufacturing company etc, then the user can check if the failure is due to sensor depreciation or due to unreliability of a specific manufacturer.

\section{Prognosis \& Diagnosis for PEMFC Systems}

In this section, initially a generic framework for semantic prognosis and diagnosis for PEMFC systems is provided; then, the description of the developed FuCSyDi platform follows. Although the focus of the current research work is on PEMFC systems, it can be extended to other fuel cell systems provided that the ontology is extended accordingly to capture their structure and the respective diagnostic rules as well.

\subsection{The Generic Framework}

The semantic prognosis process informs the user in a predefined frequency about the overall real-time performance of the system and with the use of the "evidenced" modes defined by Davies et al. (2017) is being alerted about the very early stages of any failures. The alerting mechanism is enriched with the respective explanations of the failures, allowing the user initiating mitigating actions. With the diagnosis process the user is provided with the explanations of any failures and can perform queries over them. 
Table 3: Conversion of Davies et al. (2017) rules to OWL 2 EL GCIs

\begin{tabular}{|c|c|}
\hline Rule & OWL 2 EL GCI \\
\hline 1 & $\begin{array}{l}\text { LowVoltageSensorOutput and (isValid value true) } \\
\text { SubClass Of explains value evidencedFlooding }\end{array}$ \\
\hline 2 & $\begin{array}{c}\text { LowTemperatureSensorOutput and (isValid value true) } \\
\text { SubClassOf explains value flooding }\end{array}$ \\
\hline 3 & $\begin{array}{l}\text { HighCathodeHumidityValue and (isValid value true) } \\
\text { SubClassOf explains value flooding }\end{array}$ \\
\hline 4 & $\begin{array}{l}\text { LowCathodeHumidityValue and (isValid value true) } \\
\text { SubClassOf explains value dehydration }\end{array}$ \\
\hline 5 & $\begin{array}{l}\text { HighTemperatureSensorOutput and (isValid value true) } \\
\text { SubClass Of explains value dehydration }\end{array}$ \\
\hline 6 & $\begin{array}{l}\text { LowStoichiometryValue and (isValid value true) } \\
\text { SubClassOf explains value evidencedCarbonSupportCorrosion }\end{array}$ \\
\hline 7 & $\begin{array}{l}\text { HighAnodeHumidityChangeValue and (isV alid value true) } \\
\text { SubClassOf explains value evidencedMembraneMechanicalStress }\end{array}$ \\
\hline 8 & $\begin{array}{c}\text { HighCathodeHumidityChangeValue and (isValid value true) } \\
\text { SubClass Of explains value evidencedMembraneMechanicalStress }\end{array}$ \\
\hline 9 & $\begin{array}{c}\text { isDescribedByCalculatedValue some } \\
\text { (LowAnodeHumidityChangeValue and (isValid value true)) and } \\
\text { isDescribedByCalculatedValue some } \\
\text { (LowCathodeHumidityChangeValue and }(\text { isValid value true)) } \\
\text { SubClassOf } \text { indicates value evidencedMembraneMechanicalStress }\end{array}$ \\
\hline 10 & $\begin{array}{c}\text { isDescribedByCalculatedValue some } \\
\text { (HighAnodeHumidityChangeValue and (isValid value true)) and } \\
\text { isDescribedByCalculatedValue some } \\
\text { (HighCathodeHumidityChangeValue and (isValid value true)) } \\
\text { SubClassOf } \text { indicates value membraneMechanicalStress }\end{array}$ \\
\hline 11 & $\begin{array}{l}\text { HighVoltageSensorOutput and (isValid value true) } \\
\text { SubClassOf explains value evidencedCatalystDissolution }\end{array}$ \\
\hline 12 & $\begin{array}{c}\text { hasObservedSensorMeasurement some } \\
\text { (VeryHighVoltageSensorOutput and (isValid value true)) and } \\
\text { isDescribedByCondition some LowVoltageCycleNumber) } \\
\text { SubClassOf } \text { indicates } \text { value evidencedCatalystDissolution }\end{array}$ \\
\hline 13 & $\begin{array}{c}\text { hasObservedSensorMeasurement some } \\
\text { (VeryHighVoltageSensorOutput and (isValid value true)) and } \\
\text { isDescribedByCondition some HighVoltageCycleNumber) } \\
\text { SubClassOf } \text { indicates value catalystDissolution }\end{array}$ \\
\hline 14 & $\begin{array}{c}\text { HighVoltageSensorOutput and (isValid value true) } \\
\text { SubClassOf explains value evidencedMembraneBreakdown }\end{array}$ \\
\hline 15 & $\begin{array}{l}\text { VeryHighVoltageSensorOutput and (isValid value true) } \\
\text { SubClassOf explains value membraneBreakdown }\end{array}$ \\
\hline
\end{tabular}

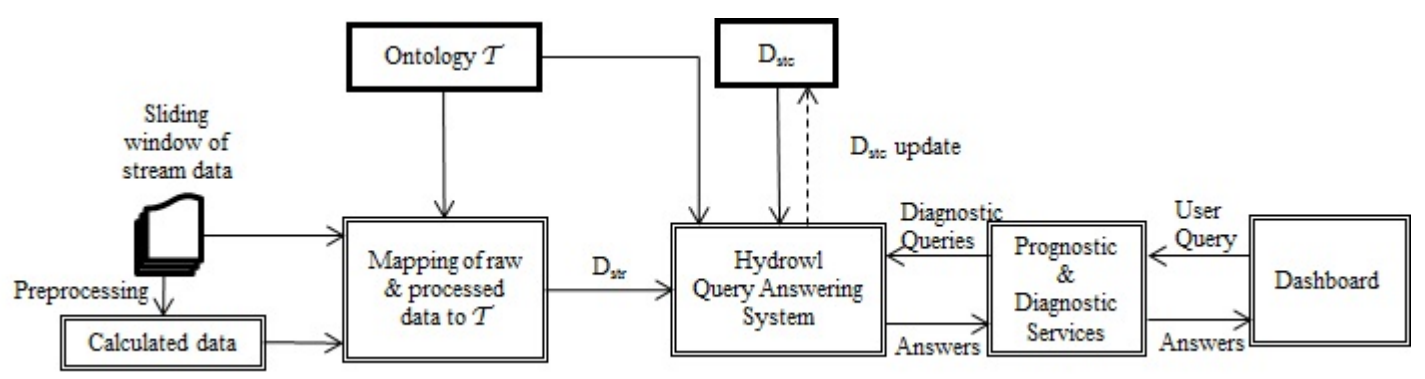

Figure 6: Generic framework for system prognosis and diagnosis 
The gathered information from the monitored system studied in this research work contains static, streaming, updated semi-static data and historical data. The static data contain the details of the technical description and the specification of the system. In particular, they are populated by assertions describing the provided information surrounding the specific fuel cell systems, their components, the sensors monitoring them, the relations among them, the scale ranges and the operation ranges of the operating conditions of the system. Additionally, in this set of data all the sensors are initially declared as reliable. The streaming data include sensor output data (temperature, voltage, etc) and the calculated data obtained from their processing (stoichiometry, humidity, humidity change), while the updated semi-static data are obtained from the automatic interrogation of the streaming and static data. Examples for updated semi-static data include the reliability of the sensors and the changing conditions of the system. The historical data constitute a portion of the old streaming data stored in the memory. The size of this portion of data is predefined by the user.

In Figure 6 the architecture of the generic proposed framework for system prognosis and diagnosis is illustrated. Initially, the user specifies the size of the sliding window that contains the historical data. Given the real-time sliding window of sensor data, the FCSM ontology and the static data $\left(D_{\text {stc }}\right)$, the following processes are performed for each stream of data:

1. Data preprocessing. This process includes the necessary calculations from the raw data that will aid the prognosis and diagnosis of the system, i.e. the calculation of the stoichiometry, of the cathode humidity and of the humidity change of the anode and the cathode catalyst layer. Also, depending the number of cells included in each stack, the voltage scale ranges are re-calculated.

2. Construction of the streaming dataset. For each time $t_{j}$, the raw and calculated data are classified according the scale ranges and mapped to the ontology, forming the streaming data $\left(\mathrm{D}_{\text {str }}\right)$. Additionally, in the $\mathrm{D}_{\text {str }}$ the state state@system ${ }_{i} t_{j}$ of each monitored system system is defined.

Example 5.1. Let $\mathrm{FCS}_{i}$ be a fuel cell stack with only one fuel cell and let $35 \mathrm{~V}$ be the voltage output of the sensor $\mathrm{s}_{\mathrm{k}}$ at the monitoring time $\mathrm{t}_{j}$. Then, the assertions in $\mathrm{D}_{\mathrm{str}}$ and $\mathrm{D}_{\mathrm{stc}}$ that are related to the voltage sensor are the following:

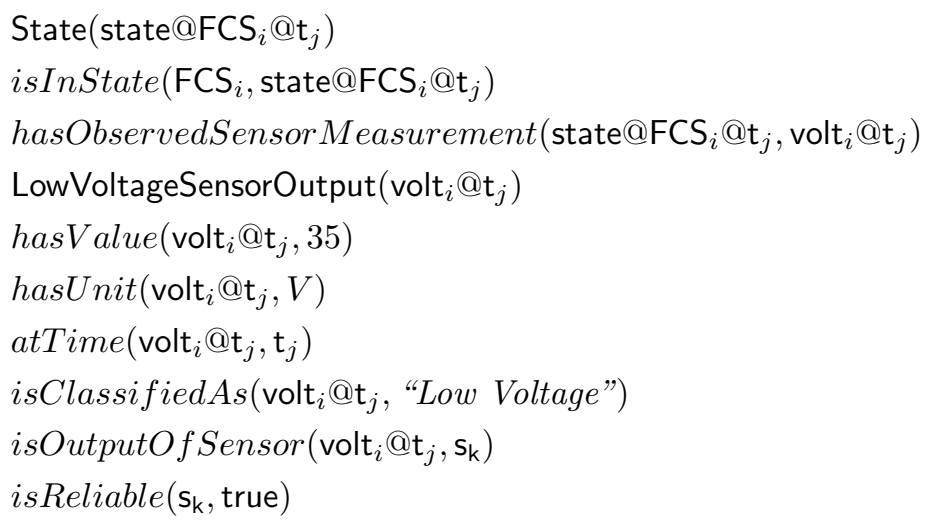

3. Knowledge inference and alerting. The ontology along with the streaming data, the static data and the semi-static data are loaded to the reasoner. During this loading process the reasoner automatically calculates all the assertions that can be inferred. Then, to perform system diagnosis it suffices to interrogate the $\mathrm{KB}$ with the following query:

$$
\begin{array}{r}
Q(x, z, v, w, m) \leftarrow \text { FuelCellStack }(x), \operatorname{isInState}(x, y), \operatorname{indicates}(y, z), \operatorname{explains}(u, z), \\
\operatorname{hasValue}(u, v), \operatorname{has} U n i t(u, w), i s C l a s s i f i e d A s(u, m)
\end{array}
$$

which returns the stacks and the degradation mode from which they suffer along with the specific values (from the sensors or calculated ones) that explain this degradation and the classification of these values. The next example illustrates the set of the calculations that the reasoner will perform automatically to return the right answers to query (18). 
Table 4: Queries for Sensor Reliability Checking

$\begin{aligned} \mathcal{Q}_{1}: Q(x, u, y, z, w) \leftarrow \quad & \text { Sensor }(x), \text { hasOutput }\left(x, x_{1}\right), \text { hasValue }\left(x_{1}, u\right), \\ & \text { hasOperatingRange }\left(x, x_{2}\right), \text { hasLowerValue }\left(x_{2}, y\right), \text { hasUpperValue }\left(x_{2}, z\right), \\ & \text { monitors }(x, w), \text { FuelCellStack }(w) \\ \mathcal{Q}_{2}: Q(x, u, y, z, m, n, w) \leftarrow \quad & \text { Sensor }(x), \text { hasOutput }\left(x, x_{1}\right), \operatorname{hasValue}\left(x_{1}, u\right), \\ & \text { hasOperatingRange }\left(x, x_{2}\right), \operatorname{hasLowerValue}\left(x_{2}, y\right), \operatorname{hasUpperValue}\left(x_{2}, z\right), \\ & \text { monitors }(x, m), \operatorname{hasPart}(n, m), \operatorname{containsFuelCell}(w, n)\end{aligned}$

Example 5.2. Let $\mathrm{D}_{\text {str }}$ and $\mathrm{D}_{\text {stc }}$ be as in Example 5.1. The reasoner infers from axiom (3) and assertion (17) that:

$$
\operatorname{isValid}\left(\operatorname{volt}_{i} @ \mathrm{t}_{j}, \text { true }\right)
$$

From (11), (19) and the first axiom of Table 3 the reasoner will infer the assertion:

$$
\text { explains }\left(\text { volt }_{i} @ \mathrm{t}_{j}\right. \text {, evidencedFlooding) }
$$

i.e. that volt $_{i} @ \mathrm{t}_{j}$ is a reason for the evidencedFlooding mode. Also, from the assertions (8), (10), (20) and the $S W R L$ rule (7) it is inferred that

$$
\text { indicates(state@FCS }{ }_{i} @ \mathrm{t}_{j} \text {, evidencedFlooding) }
$$

Hence, from the assertions (8), (12), (13), (15), (20), (21), the following tuple:

$\left\{\mathrm{FCS}_{i}\right.$, evidencedFlooding, $35, V$, "Low Voltage" $\}$

will be computed as the answer to query (18).

4. Sensor reliability checking. At the same time, the sensor outputs are compared to their respective operation values by performing the queries $\mathcal{Q}_{1}, \mathcal{Q}_{2}$ presented in Table 4 . Query $\mathcal{Q}_{1}$ is for sensors that monitor parameters related to the stack in general, such as the voltage or the current sensors, while query $\mathcal{Q}_{2}$ is for sensors monitoring the behaviour of particular parts of the system, like the humidity sensor. In particular, when query $\mathcal{Q}_{1}$ is performed, the reasoner will return all the sensors along with their output value at the monitoring moment, the stacks they are monitoring and their operation ranges. Similarly, with query $\mathcal{Q}_{2}$ the reasoner will return the same results but besides the monitored stack it will return the exact part of the stack that each sensor monitors. If the output values of a particular sensor $\mathrm{s}_{\mathrm{i}}$ violates its operation ranges for $x$ consecutive states, where $x$ the size of the sliding window predefined by the user, then $s_{i}$ is defined as unreliable. Hence, the dataset $D_{\text {stc }}$ is updated accordingly and the diagnostic system informs the user that the sensor $s_{i}$ that monitors the specific (part of the) stack is unreliable. Once a sensor is defined as unreliable then this information does not change. However, if a sensor is defined as reliable then it is continuously checked with respect to its reliability throughout the time of operation of the system.

At this point, it is important to note that the reliability checking algorithm that is proposed constitutes only a first approach to this problem. More complex and accurate algorithms have been suggested in the literature, e.g. (Mao et al., 2018), which will be employed in future work.

5. Sliding the window of data. The first line of the window of data is replaced with the stream of new real-time data. This way, a moving window of data of fixed size is formed in a last-in-first-out manner. The platform proceeds by repeating the steps 1 to 5 with the new data, until the fuel cell system is stopped. It is important to note that at step 3 the user is alerted about some abnormal behaviour of the system, only if this behaviour has changed from the one in the previous state.

Additionally, during the operation of the system, the user can interrogate the KB. For instance, the user can be informed about the ambient temperature:

$$
Q(x, t) \leftarrow \operatorname{inCondition}(x, t), \text { AmbientTemperature }(t)
$$




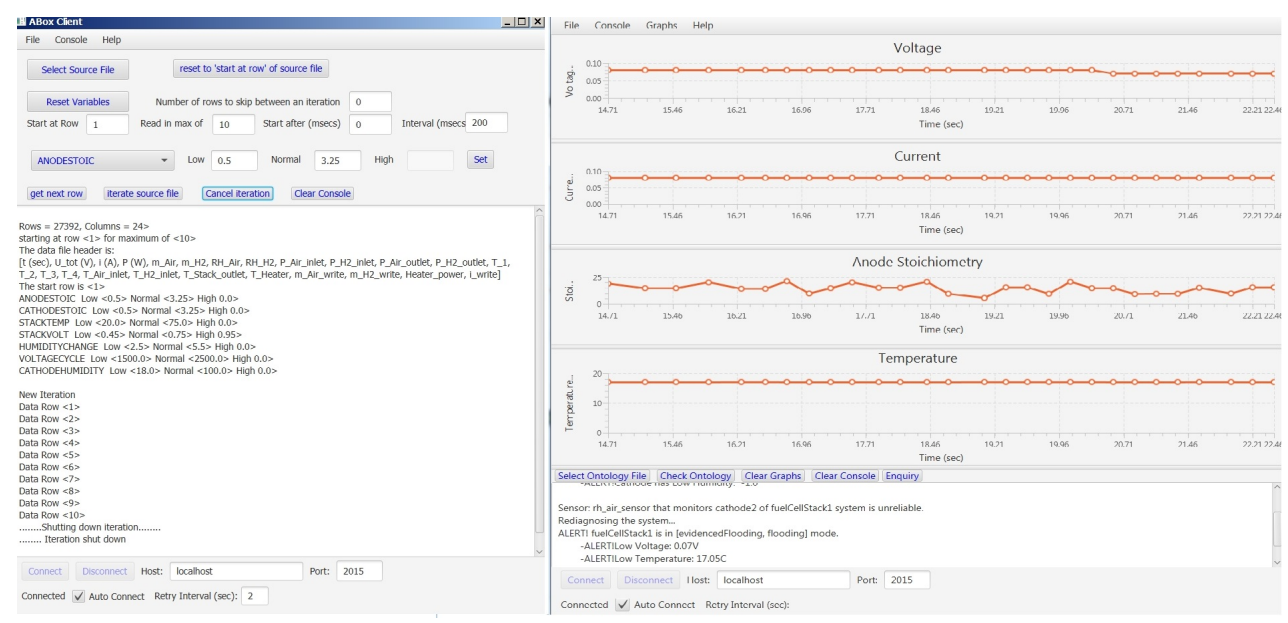

Figure 7: The FuCSyDi platform interface. The client appears on the left side and the server on the right side of the picture. In this example, the client is set to iterate 10 lines of data at a rate of $200 \mathrm{~ms}$ per line of sensor data.

or about the outputs of the sensors monitoring a particular stack (e.g. $\mathrm{FCS}_{\mathrm{i}}$ ) of the system at a specific moment (e.g. on the $30^{\text {th }}$ of October, 2018, at 14:17:52):

$Q(x) \leftarrow$ isInState $\left(\mathrm{FCS}_{\mathrm{i}}, s\right)$, hasObservedSensor Measurement $(s, x)$, atTime $(x, 2018-10-30 \mathrm{~T} 14: 17: 52)$

It is important to note though that the user can access only the part of the historical data within the sliding window of data.

\subsection{The Application}

The FuCSyDi platform consists of a client-server system written in Java 1.8 and JavaFX.

The client screen is illustrated on the left of Figure 7. The user loads the data from the sensors in the client and simulates the streaming of data. Currently, the data is captured from source (e.g. lab experiment generated or simulated data) and supplied to the client. The user can control from the client how quickly the next set of sensor data is passed to the server, whether it is the next streaming line or a specified number of lines after the last line read and the size of the sliding window of data. Finally, although the client loads the default scale range values for each of the parameters (temperature, stoichiometry, etc) automatically, the user is also allowed to reset them.

The server loads the ontology, the static data, the sensor data received from the client and constructs the streaming dataset $D_{\text {str }}$. The server screen is illustrated on the right side of Figure 7 . It has three main functionalities: 1) provides a graphical representation of the performance of the system through time (in seconds). In particular, it displays four graphs showing the status of four key metrics: the current (A), the voltage $(\mathrm{V})$, the temperature $\left({ }^{\circ} \mathrm{C}\right)$ and the stoichiometry of the anode; 2) performs on the fly the diagnostic processes described in Section 5.1. More specifically, it alerts the operator about any occurring or forthcoming failure and indicates the specific reasons underpinning this failure; 3) allows the operator to interrogate the knowledge base while the diagnostic process is running. For instance, in Figure 8 a query about the temperature of the stack and the ambient temperature for the last ten measurements (which is the size of the sliding window defined initially by the operator) is posed to the platform. The set of returned answers appears underneath the query. In particular, each bracket includes: the name of the sensor; the stack temperature along with its unit; the ambient temperature along with its unit and the time that they were monitored. 


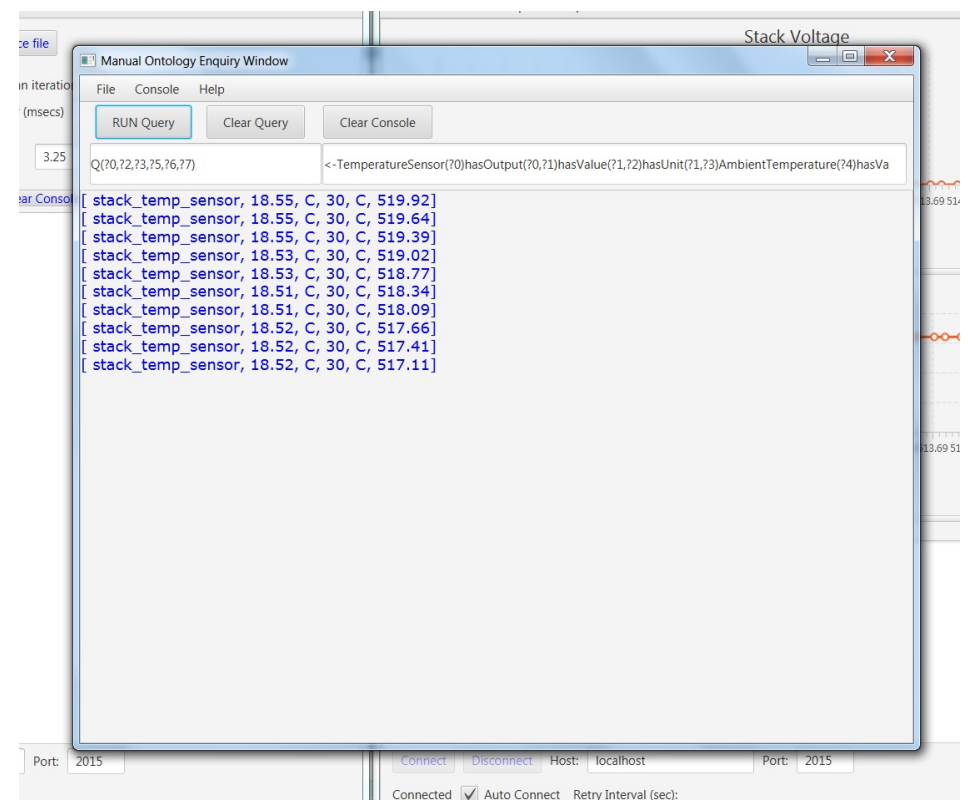

Figure 8: The query answering service

\section{Evaluation}

For the validation of the FuCSyDi platform three different experiments were performed which resulted in water management issues, as water management is one of the most critical issues for high-performance PEMFCs (Nguyen \& Knobbe, 2003). One of the key reasons for this is that they can evolve in a few seconds and can cause complete degradation of the system. As, according to experimental experience, both the flooding and dehydration reduce the voltage output, it can be difficult to distinguish between the two, hence preventing the operator from performing the suitable mitigating action.

The experiments where conducted by using new $100 \mathrm{~cm}^{2}$ PRO-RD cells manufactured by Pragma Industries. Each stack uses established materials in its construction; Nafion-XL $25 \mu \mathrm{m}$ thick polymer membrane, platinum on carbon black catalyst of $0.2 \mathrm{mg} / \mathrm{cm} 2$ loading, Sigracet 10 BC carbon paper GDE, with PTFE coating, Graphtek LLC GR-940 graphite composite monopolar plates, with 7-fold serpentine through-flow gas channels and three-fold serpentine water-coolant channels, silicone gaskets, gold plated current collectors and anodised aluminium end-plates.

All experimental settings were such that the flow rate, pressure, temperature, and humidification supply to the anode and the cathode could be controlled and monitored via the National Instruments LabVIEW programme. Also, the water circuit through the fuel cell could be cooled or heated to control temperature. In all cases normal performance was first established through steady-state operation. Then, flooding or dehydration was triggered by changing the temperature, the current density or the humidification of the system. All experiments were conducted on a single stack voltage cycle.

The data from the sensors were accessed by the application's client program. Each streaming line equates to the sensors' output collected at a specific interval. The time interval between two consecutive measurements was approximately $200 \mathrm{~ms}$. The sliding window was defined to be of size 10 rows.

The evaluation was conducted on an Intel(R) Core (TM) i5 $2.40 \mathrm{GHz}$ PC running Windows 7 with $8 \mathrm{~GB}$ of RAM.

\subsection{First Experiment: Two-Cell Stack in Flooding}

A two cell PEMFC stack was used to carry out the first experiment. In this experiment the flooding states were triggered by changing the current so that water condensation was favoured. As soon as some flooding appeared the operators acted to prevent the complete breakdown of the system. 
Voltage (V)

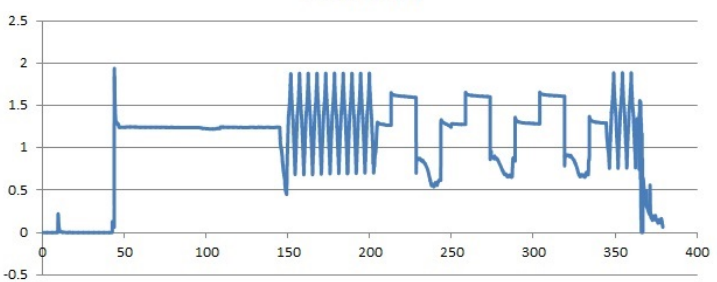

Temperature $\left({ }^{\circ} \mathrm{C}\right)$

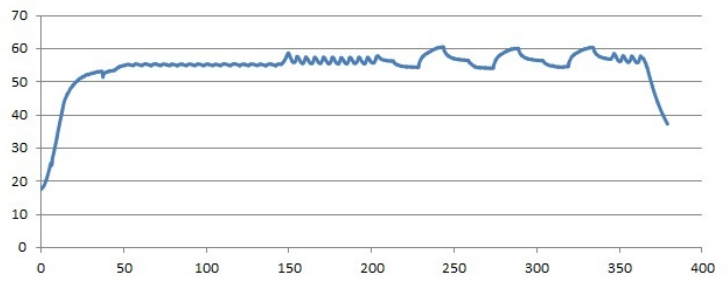

Current (A)

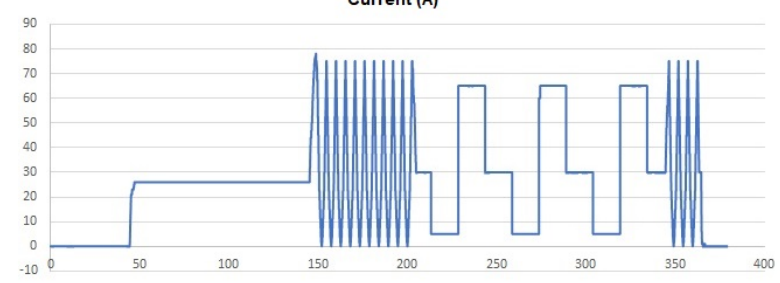

Figure 9: First Experiment: Voltage and temperature progression through time (min.).

Figure 9 demonstrates the performance of the voltage and the changes of the current and the temperature through time. A steady state performance was established (after the polarization curves) at approximately the $204^{t h}$ minute. From that moment until the $213^{\text {rd }}$ minute the fuel cell system remains in a steady mode (first phase). After this, the current is decreased from $25 \mathrm{~A}$ to $5 \mathrm{~A}$ causing voltage increase from $1.3 \mathrm{~V}$ to approximately $1.6 \mathrm{~V}$ until the $228^{\text {th }}$ minute (second phase), when the current is increased to $65.1 \mathrm{~A}$ and the voltage is instantly dropped to $0.7 \mathrm{~V}$. After this, the current remains constant for 15 minutes (third phase) and the voltage decreases to $0.5 \mathrm{~V}$, after some variations. After that point, the system recovers and the same sequence of events is repeated two more times. The variations of the temperature during these three phases are within the normal limits, hence they are insignificant.

Figures 9, 10 and 11 illustrate the output of the platform during these three phases. It is important to note that although the variations of the current and the voltage can be clearly observed in the figures, the temperature variations -which occur in a range of 7 degrees- are not visible due to the scaling of the temperature plot. During the first phase, the platform notified the operator that the relative humidity sensor $\mathrm{rh}_{\mathrm{air}}$ is unreliable, as its output values for the first 10 consecutive measurements were violating the operating range thresholds. In many cases the sensor output exceeded its high threshold by $120 \%$. The diagnostic process continued without taking into consideration the $\mathrm{rh}_{\mathrm{air}}$ sensor and the outcome was that the system was in the healthy mode. During the second phase the system is found to be under two failure modes: evidenced catalyst dissolution and evidenced membrane breakdown. The reasoner infers these two failures due to the high voltage $(>1.5 \mathrm{~V})$ and the rules 11 and 14 of Table 1 . During the third phase the platform alerts the operator that the system is in the evidenced flooding mode. According to Table 1 , an indication of this mode is the low voltage $(<0.9 \mathrm{~V})$.

\subsection{Second Experiment: Single-Cell Stack in Flooding}

In this experiment a single-cell stack was used. Flooding was induced by operating at high current loading at normal temperature $\left(50-55^{\circ} \mathrm{C}\right)$.

In Figure 12 the voltage performance, the temperature, and the current time histories are shown. The experiment is separated in three phases based on the performance of the current. The first phase starts after a relatively steady performance is stabilised, which occurs at the $14^{\text {th }}$ minute and lasts for approximately 60 minutes, right until the first polarizarion curve. The second phase is characterized by the timeframe between the two polarization curves $\left(100^{t h}-119^{t h}\right.$ minute) and the third phase starts after the second polarization curve (138 ${ }^{\text {th }}$ minute) and ends before the third polarization curve ( $285^{\text {th }}$ minute). The platform is not tested during the polarization curves as they do not correspond to an expected behaviour of a realistic application. 


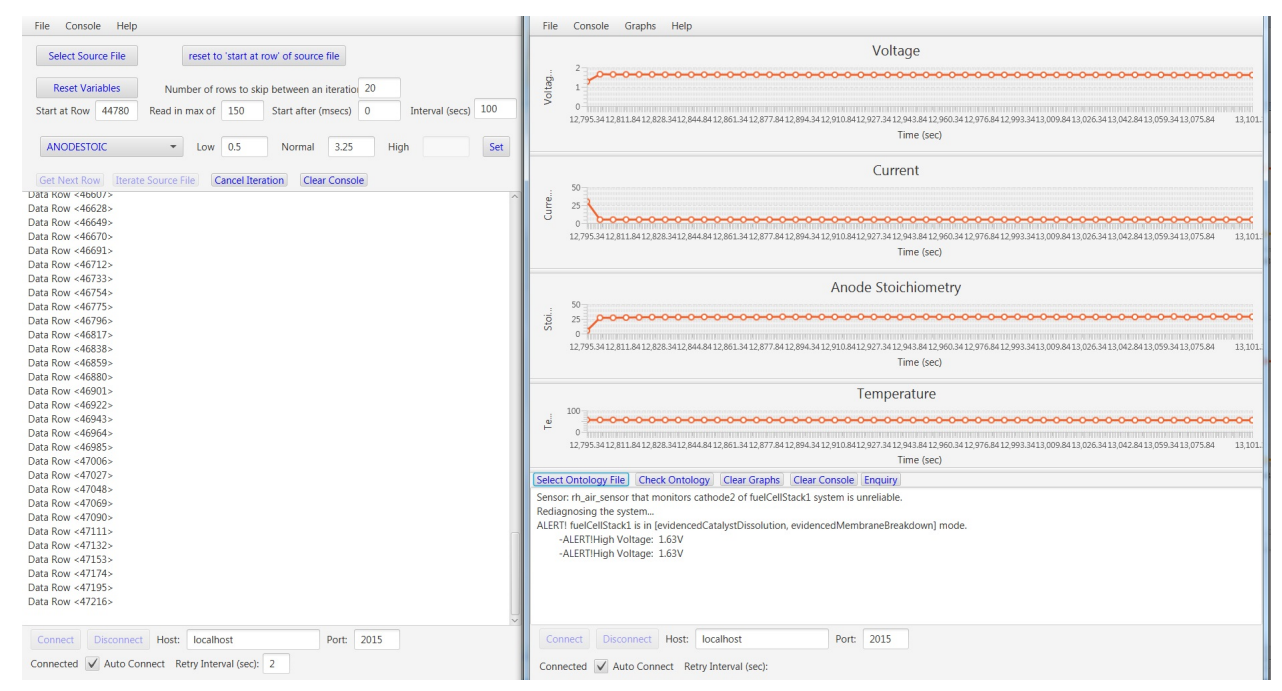

Figure 10: First Experiment: Client and server screen during the second phase.

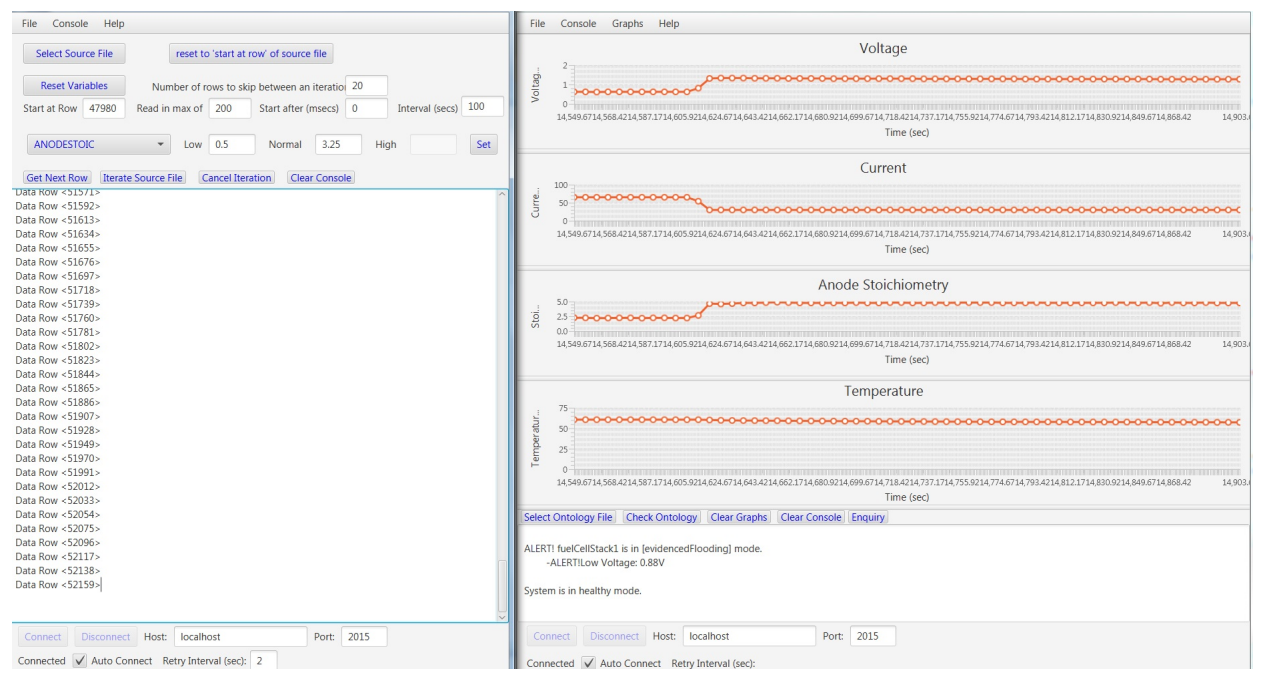

Figure 11: First Experiment: Client and server screen during the third phase. 

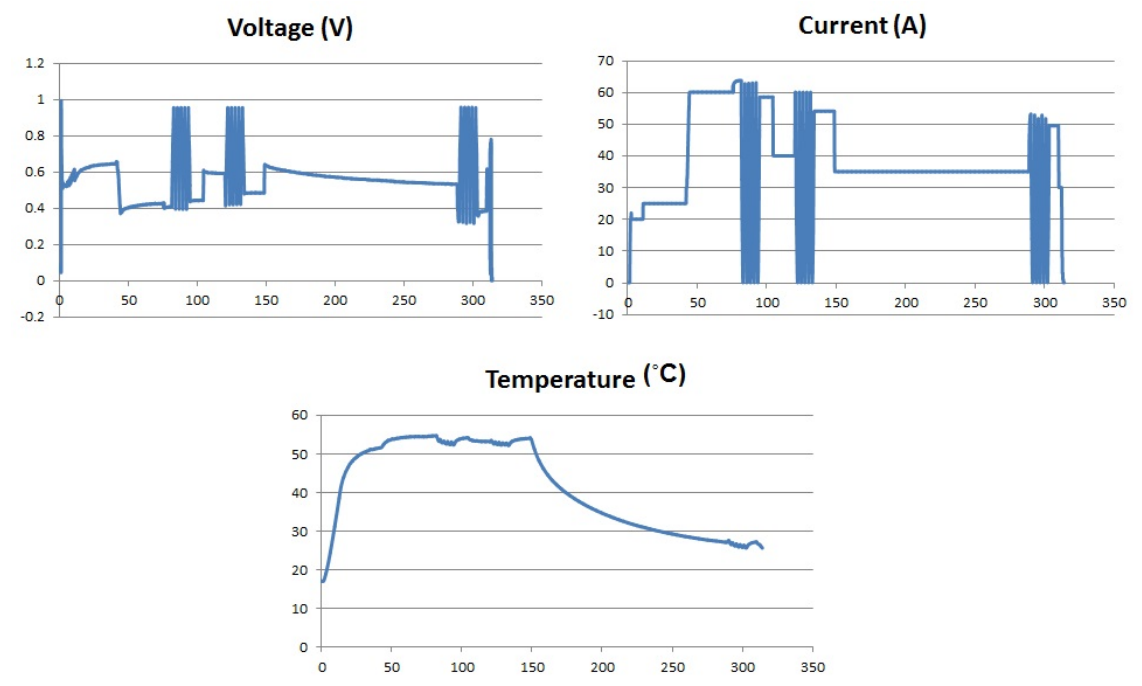

Figure 12: Second Experiment: Voltage, current and temperature progression through time (min.).

Figures 13, 14 and 15 illustrate the output of the platform during the three phases. During the first 30 minutes of the first phase the platform indicates that the system is in the healthy mode. Then, at the $44^{t h}$ minute and onwards the platform alerts the end-user that flooding possibly is occurring, as the voltage is low $(<0.45 \mathrm{~V})$. This inference is based on the first rule of Table 1. Similarly, during the beginning of the second phase the system is found to be under the "evidenced flooding" mode. Again, the low voltage is the indicator of this mode. After approximately the $104^{t h}$ minute the platform indicates that fuel cell system is back in the healthy mode. Finally, throughout the third phase the platform shows that the system is in the healthy mode.

\subsection{Third Experiment: Single-Cell Stack in Dehydration}

In this experiment the dehydration was triggered by operating the system at open circuit voltage (OCV) without any humidification. The voltage, current and temperature time histories are illustrated in Figure 16. The deviations appearing before the $36^{\text {th }}$ minute, where the dehydration was initiated, are due to the operator's actions to operate the fuel cell at OCV. According to experimental experience, the rapid decrease of the cell voltage that appears after the $36^{\text {th }}$ minute indicates that some water management issue is occurring. However, as the behaviour of the temperature remains normal it is hard to indicate whether it is flooding or dehydration.

The Figure 17 illustrates the output of the platform until the $68^{\text {th }}$ minute (after this minute the behaviour of the stack is relatively stable until the appearance of the polarization curves and the eventual shutting down of the system). It is interesting to note that, as in the previous examples, the relative humidity sensors are identified as unreliable. Hence, the occurring dehydration cannot be directly derived from the $4^{\text {th }}$ rule of Table 1 and thus, it is not diagnosed at all. Instead, as in the second phase, the platform diagnosed some catalyst dissolution and some membrane chemical breakdown.

\subsection{Discussion}

The platform diagnosed successfully the failure modes of the first two experiments and it failed to diagnose the dehydration occurring during the third experiment. In the first two experiments the prognostic nature of the platform is observed, as in both cases the evidenced flooding mode is indicated at a very early stage, thus long before the degradation of the stack. Indeed, in both cases, traces of water where observed by the operators of the experiment. 


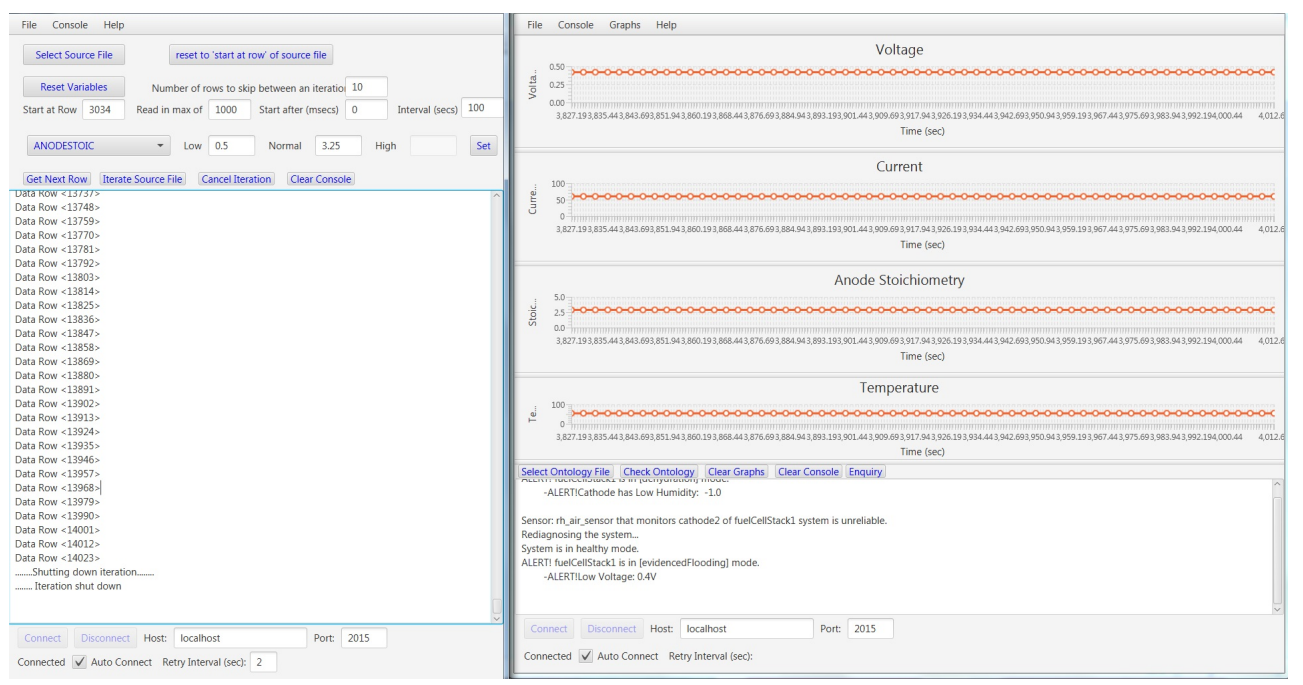

Figure 13: Second Experiment: Client and server screen during the first phase.

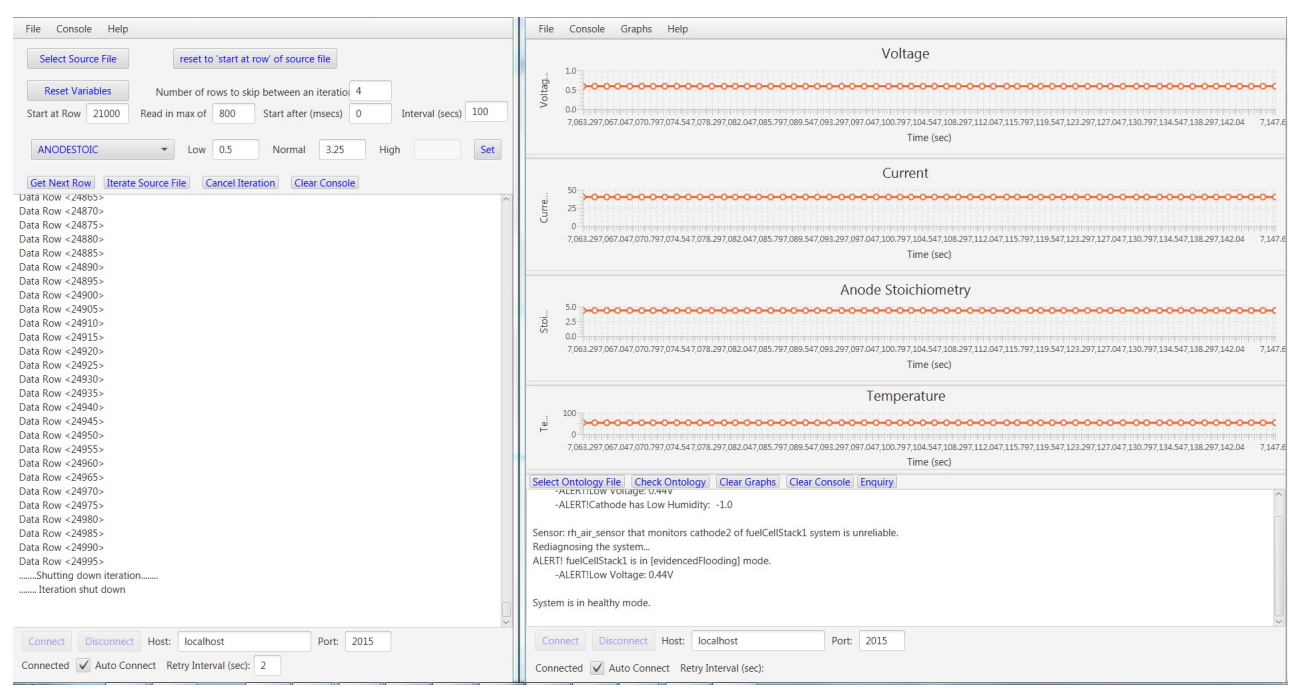

Figure 14: Second Experiment: Client and server screen during the second phase. 


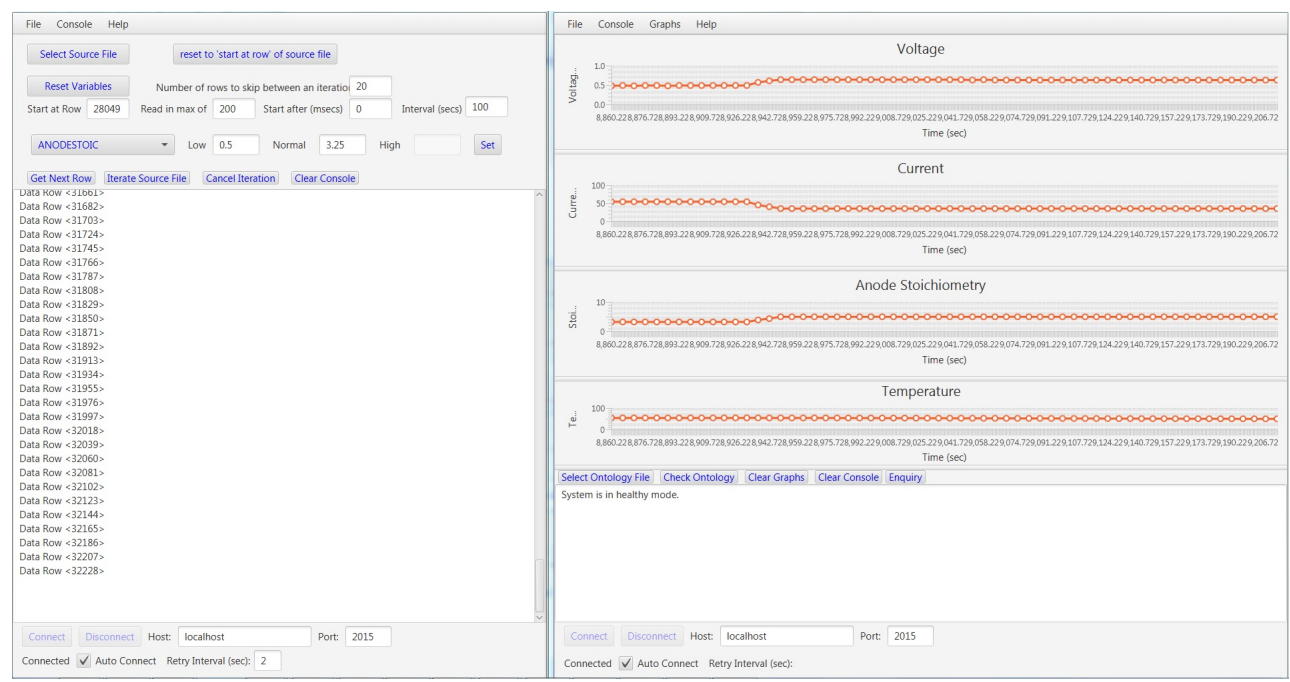

Figure 15: Second Experiment: Client and server screen during the third phase.
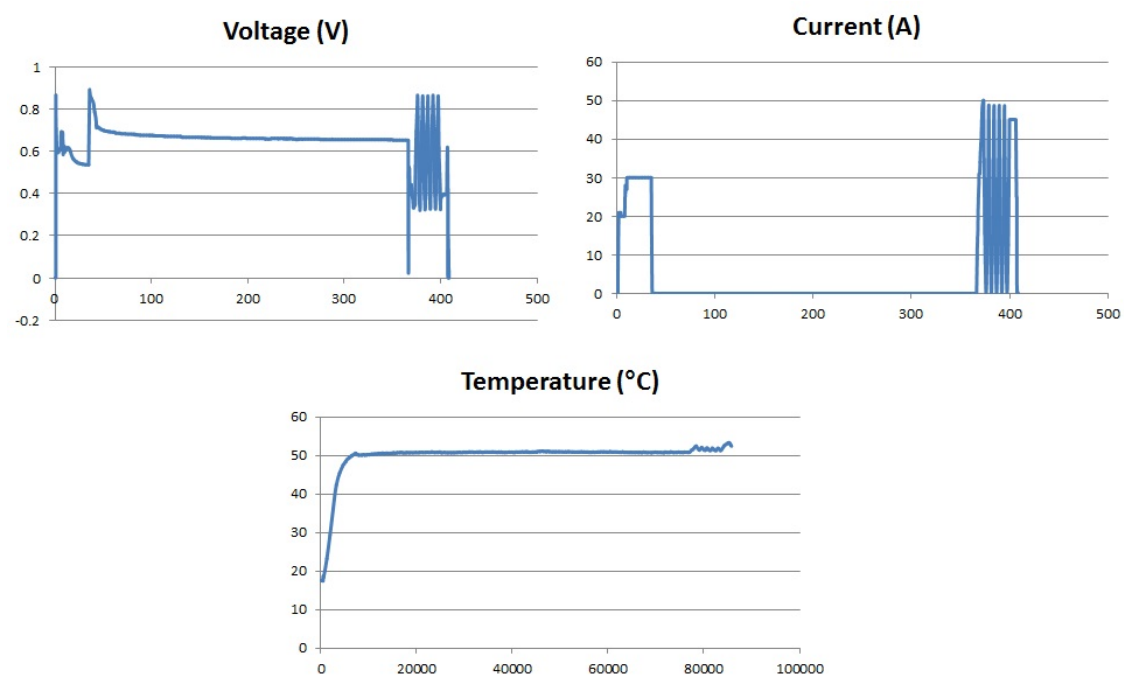

Figure 16: Third Experiment: Voltage, current and temperature progression through time (min.). 


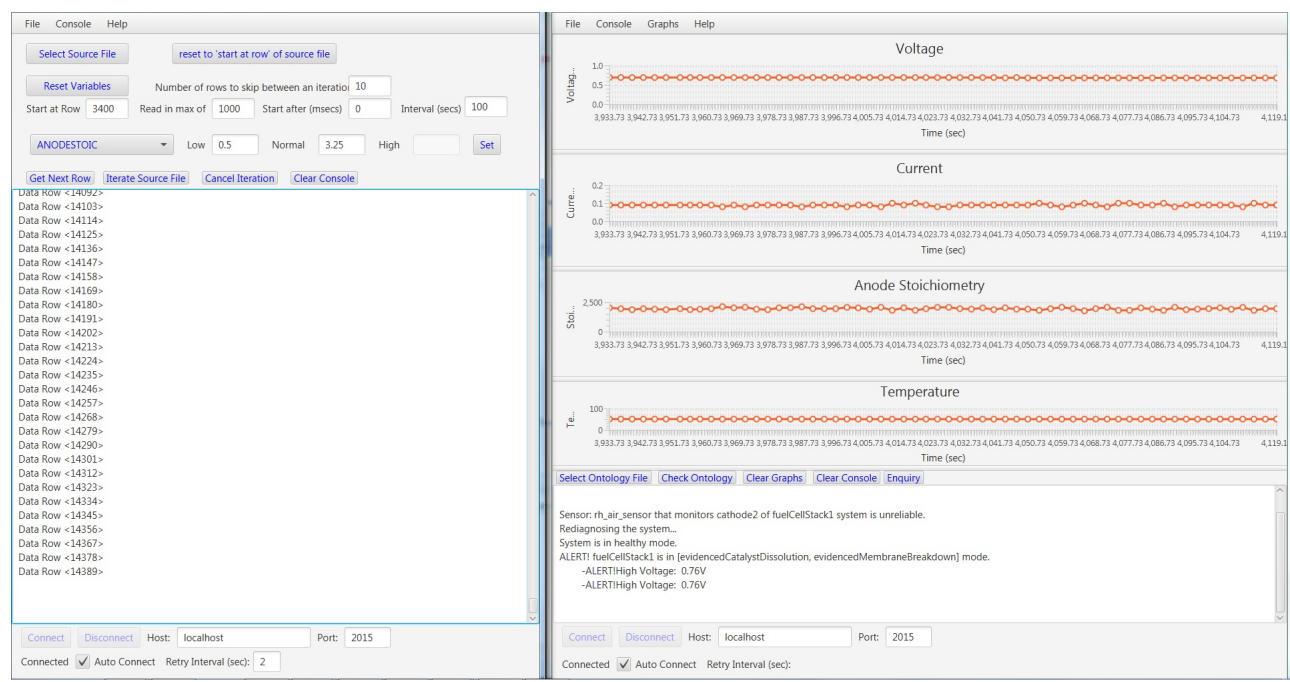

Figure 17: Third Experiment: Client and server screen.

Although the low humidity of the system in the third experiment can explain its dehydration, this cannot be witnessed from the platform, as the relative humidity sensors are indicated as unreliable and hence are outputs are discarded. This diagnostic issue could be overcame if the platform was equipped with an external mechanism indicating the abrupt drop of the voltage and the ontology was extended with a new rule stating that this bahaviour of the voltage implies that there is some water management issue. In that case, given that the voltage is not low, thus according to the $1^{\text {st }}$ rule of Table 1 the issue is not flooding, the platform would automatically infer that the stack is suffering from dehydration.

\section{Conclusion}

In this research a semantic technology-based platform for early diagnosis of PEM fuel cell systems has been proposed. The aim of this work was to provide a user friendly application environment that facilitates the real-time identification and diagnosis of potential issues, deterioration and breakdown within a fuel cell system.

The novel Fuel Cell System Monitoring ontology was introduced to serve as a semantic layer through which the end-user is provided with access to the knowledge implied from the raw data and to form the ground for a reasoning based diagnostic mechanism enriched with semantic context. The ontology contains all the information related to the structure of the PEMFC systems along with the diagnostic rules used. The semantic context of the FCSM ontology was further enhanced by linking it to the SOSA ontology which is endorsed by the W3C community. On top of the ontology, the lightweight FuCSyDi platform was developed. The FuCSyDi platform alerts the end-user about any forthcoming or occurring failures indicating the reasons for these failures and provides an overview of the fuel cell system performance through demonstrated plots, while, at the same time, is enabled to interrogate the knowledge base for further analysis. It is important to emphasize that, in contrary to the most current diagnostic systems, the FuCSyDi platform automatically detects any faulty sensors and isolates their outputs during the diagnostic process.

The validity of the platform was tested using three different experimental settings. The platform diagnosed instantly the occurring failure of the first two experiments. However, the evaluation results of the third experiment showed that the ontology should be extended with new diagnostic rules to capture dehydration. Dealing with multiple failure modes and their interaction effects is still an ongoing topic of research, with developments in this area new rules need to be generated and added to increase the capability of the FuCSyDi platform. At the same time, the abrupt changes in the performance of the system, should also be used for diagnosis as they may imply some degradation of the system. Another limitation of the current 
form of the FuCSyDi platform is that it does not take under consideration the duration of an abnormal behavior of the system, leading to possibly fault diagnostic results, due to outliers. Finally, the reliability checking of the sensors is limited to ensuring that they operate within their normal operating ranges, thus other cases where the sensor outputs of the system may indicate a faulty sensor are not captured yet.

The dynamic nature of the semantic technologies enables an easy path for expanding the incorporated knowledge. Hence, future research should seek to introduce new diagnostic rules to enhance the diagnostic performance of the FuCSyDi platform, by taking also under consideration the degradation effects of cumulative faults, long-term storage, start-stop cycling and environmental conditions. Evaluating the impact of new sensors to enhance diagnosability is an area for future research, where the results would impact directly on new diagnostic rules which could be added to further differentiate between differing modes. Additionally, we are planning to employ temporal ontology reasoning techniques (Batsakis et al., 2017) to capture possible outliers and abrupt changes of system's performance, or seek other techniques that can detect possible outliers. By employing fuzzy ontological languages (Bobillo \& Straccia, 2011) and fuzzy reasoning techniques (Bobillo \& Straccia, 2016) the fuzzy PEMFC diagnostic rules appearing in the literature will be captured more accurately, enhancing the performance of the FuCSyDi platform. To further improve the accuracy of the platform's results, we plan to adopt more sophisticated sensor-reliability-checking algorithms. The operational validity of such sensors is key to the performance of the system. Finally, a long-term goal is to extend FuCSyDi's functionality to different types of fuel cells as well and to be enriched with rules that will aid the automatic suggestion of the appropriate mitigation action.

Overall, this research has identified an innovative approach that can tackle some of the barriers to the adoption of fuel-cell systems, namely detailed early fault detection and intervention management of the system.

\section{Acknowledgement}

This work was supported by the UK Engineering and Physical Sciences Research Council (EPSRC) for Loughborough University (grants EP/K014137/1 and EP/K02101X/1).

\section{References}

Andreasen, S. J., Vang, J. R., \& Kær, S. K. (2011). High temperature PEM fuel cell performance characterisation with co and co2 using electrochemical impedance spectroscopy. International journal of hydrogen energy, 36, 9815-9830.

Antoniou, G., Groth, P., Harmelen, F., \& Hoekstra, R. (2012). A Semantic Web Primer. The MIT Press.

Asensio, F., Martin, J. S., Zamora, I., \& Garcia-Villalobos, J. (2017). Fuel cell-based chp system modelling using artificial neural networks aimed at developing techno-economic efficiency maximization control systems. Energy, 123 , 585 - 593. URL: http://www.sciencedirect.com/science/article/pii/S0360544217302177. doi:https://doi.org/10.1016/j. energy.2017.02.043.

Atkinson, R., García-Castro, R., Lieberman, J., \& Stadler, C. (2017). Semantic Sensor Network Ontology. W3C Working Draft. Available at https://www.w3.org/TR/vocab-ssn/.

Baader, F., Brandt, S., \& Lutz, C. (2005). Pushing the EL envelope. In Proceedings of the 19th International Joint Conference on Artificial Intelligence IJCAI'05 (pp. 364-369). San Francisco, CA, USA: Morgan Kaufmann Publishers Inc.

Baader, F., Calvanese, D., McGuinness, D. L., Nardi, D., \& Patel-Schneider, P. F. (Eds.) ((2003)). The Description Logic Handbook: Theory, Implementation, and Applications. New York, NY, USA: Cambridge University Press.

Batsakis, S., Petrakis, E. G., Tachmazidis, I., \& Antoniou, G. (2017). Temporal representation and reasoning in owl 2. Semantic Web, 8, 981-1000.

Bishop, B., Kiryakov, A., Ognyanoff, D., Peikov, I., Tashev, Z., \& Velkov, R. (2011). OWLIM: A family of scalable semantic repositories. Semantic Web, 2, 33-42.

Bobillo, F., \& Straccia, U. (2011). Fuzzy ontology representation using OWL 2. International Journal of Approximate Reasoning, 52, 1073-1094.

Bobillo, F., \& Straccia, U. (2016). The fuzzy ontology reasoner fuzzyDL. Knowledge-Based Systems, 95, 12-34.

Borup, R., Meyers, J., Pivovar, B., Kim, Y. S., Mukundan, R., Garland, N., Myers, D., Wilson, M., Garzon, F., Wood, D., Zelenay, P., More, K., Stroh, K., Zawodzinski, T., Boncella, J., McGrath, J. E., Inaba, M., Miyatake, K., Hori, M., Ota, K., Ogumi, Z., Miyata, S., Nishikata, A., Siroma, Z., Uchimoto, Y., Yasuda, K., Kimijima, K.-i., \& Iwashita, N. (2007). Scientific aspects of polymer electrolyte fuel cell durability and degradation. Chemical Reviews, $107,3904-3951$. doi:10.1021/cr0501821.

Chung, C. G., Kim, L., Sung, Y. W., Lee, J., \& Chung, J. S. (2009a). Degradation mechanism of electrocatalyst during long-term operation of PEMFC. International Journal of Hydrogen Energy, 34, 8974-8981. 
Chung, C. G., Kim, L., Sung, Y. W., Lee, J., \& Chung, J. S. (2009b). Degradation mechanism of electrocatalyst during long-term operation of PEMFC. International Journal of Hydrogen Energy, 34, 8974 - 8981. doi:https://doi.org/10. 1016/j.ijhydene.2009.08.094.

Compton, M., Barnaghi, P., Bermudez, L., García-Castro, R., Corcho, O., Cox, S., Graybeal, J., Hauswirth, M., Henson, C., Herzog, A., Huang, V., Janowicz, K., Kelsey, W. D., Le Phuoc, D., Lefort, L., Leggieri, M., Neuhaus, H., Nikolov, A., Page, K., Passant, A., Sheth, A., \& Taylor, K. (2012). The SSN ontology of the W3C Semantic Sensor Network Incubator Group. Web Semant., 17, 25-32.

Damour, C., Benne, M., Grondin-Perez, B., Bessafi, M., Hissel, D., \& Chabriat, J.-P. (2015). Polymer electrolyte membrane fuel cell fault diagnosis based on empirical mode decomposition. Journal of Power Sources, 299, 596-603.

Davies, B., Jackson, L., \& Dunnett, S. (2017). Expert diagnosis of polymer electrolyte fuel cells. International Journal of Hydrogen Energy, 42, 11724 - 11734.

Eslamloueyan, R. (2011). Designing a hierarchical neural network based on fuzzy clustering for fault diagnosis of the tennesseeeastman process. Applied soft computing, 11, 1407-1415.

Fecarotti, C., Andrews, J., \& Chen, R. (2016). A petri net approach for performance modelling of polymer electrolyte membrane fuel cell systems. International Journal of Hydrogen Energy, 41, 12242-12260.

Ferreira, P., Shao-Horn, Y., Morgan, D., Makharia, R., Kocha, S., Gasteiger, H. et al. (2005). Instability of pt/ c electrocatalysts in proton exchange membrane fuel cells a mechanistic investigation. Journal of The Electrochemical Society, 152, A2256A2271.

Glimm, B., Horrocks, I., Motik, B., Stoilos, G., \& Wang, Z. (2014). HermiT:an OWL 2 reasoner. Journal of Automated Reasoning, 53, 245-269.

He, Q. P., Qin, S. J., \& Wang, J. (2005). A new fault diagnosis method using fault directions in fisher discriminant analysis. AIChE journal, 51, 555-571.

Hitzler, P., Krtzsch, M., Parsia, B., Patel-Schneider, P. F., \& Rudolph, S. (2009). OWL 2 Web Ontology Language Primer. W3C Recommendation World Wide Web Consortium. URL: http://www.w3.org/TR/ow12-primer/.

Horridge, M., \& Patel-Schneider, P. F. (2008). Manchester syntax for OWL 1.1. In OWLED (Spring).

Horrocks, I., Patel-Schneider, P. F., Boley, H., Tabet, S., Grosofand, B., \& Dean, M. (2004). SWRL: A semantic web rule language combining OWL and RuleML. W3C Member Submission.

Inaba, M., Kinumoto, T., Kiriake, M., Umebayashi, R., Tasaka, A., \& Ogumi, Z. (2006). Gas crossover and membrane degradation in polymer electrolyte fuel cells. Electrochimica Acta, 51, 5746-5753.

Jeppesen, C., Araya, S. S., Sahlin, S. L., Thomas, S., Andreasen, S. J., \& Kær, S. K. (2017). Fault detection and isolation of high temperature proton exchange membrane fuel cell stack under the influence of degradation. Journal of Power Sources, $359,37-47$.

Jha, M., Bressel, M., Ould-Bouamama, B., \& Dauphin-Tanguy, G. (2016). Particle filter based hybrid prognostics of proton exchange membrane fuel cell in bond graph framework. Computers \& Chemical Engineering, 95, $216-230$.

Kabza, A. (2016). Just another Fuel Cell Formulary. Technical Report. URL: http://www.pemfc.de/FCFSmart.pdf.

Kamal, E., \& Aitouche, A. (2018). Fuzzy observer-based fault tolerant control against sensor faults for proton exchange membrane fuel cells. International Journal of Hydrogen Energy, . doi:10.1016/j.ijhydene.2018.10.070.

Khan, M. J., \& Iqbal, M. T. (2005). Modelling and analysis of electro-chemical, thermal, and reactant flow dynamics for a PEM fuel cell system. Fuel Cells, 5, 463-475. doi:10.1002/fuce.200400072.

Kharlamov, E., Hovland, D., Jiménez-Ruiz, E., Lanti, D., Lie, H., Pinkel, C., Rezk, M., Skjæveland, M. G., Thorstensen, E., Xiao, G., Zheleznyakov, D., \& Horrocks, I. (2015). Ontology Based Access to Exploration Data at Statoil. In M. Arenas, O. Corcho, E. Simperl, M. Strohmaier, M. d'Aquin, K. Srinivas, P. Groth, M. Dumontier, J. Heflin, K. Thirunarayan, \& S. Staab (Eds.), Proceedings of the International Semantic Web Conference, The Semantic Web, ISWC'15 (pp. 93-112). Cham: Springer International Publishing.

Kharlamov, E., Mailis, T., Mehdi, G., Neuenstadt, C., zgr zep, Roshchin, M., Solomakhina, N., Soylu, A., Svingos, C., Brandt, S., Giese, M., Ioannidis, Y., Lamparter, S., Mller, R., Kotidis, Y., \& Waaler, A. (2017). Semantic access to streaming and static data at siemens. Web Semantics: Science, Services and Agents on the World Wide Web, 44, 54-74.

Kharlamov, E., Solomakhina, N., Özçep, Ö. L., Zheleznyakov, D., Hubauer, T., Lamparter, S., Roshchin, M., Soylu, A., \& Watson, S. (2014). How semantic technologies can enhance data access at siemens energy. In P. Mika, T. Tudorache, A. Bernstein, C. Welty, C. Knoblock, D. Vrandečić, P. Groth, N. Noy, K. Janowicz, \& C. Goble (Eds.), The Semantic Web - ISWC 2014 (pp. 601-619). Cham: Springer International Publishing.

Kheirandish, A., Shafiabady, N., Dahari, M., Kazemi, M. S., \& Isa, D. (2016). Modeling of commercial proton exchange membrane fuel cell using support vector machine. International Journal of Hydrogen Energy, 41, 11351-11358.

Larminie, J., Dicks, A., \& McDonald, M. (2003). Fuel Cell Systems Explained volume 2. J. Wiley Chichester, UK.

Lécué, F., Schumann, A., \& Sbodio, M. L. (2012). Applying semantic web technologies for diagnosing road traffic congestions. In P. Cudré-Mauroux, J. Heflin, E. Sirin, T. Tudorache, J. Euzenat, M. Hauswirth, J. X. Parreira, J. Hendler, G. Schreiber, A. Bernstein, \& E. Blomqvist (Eds.), Proceedings of the International Semantic Web Conference, The Semantic Web, ISWC'12, (pp. 114-130). Berlin, Heidelberg: Springer Berlin Heidelberg.

Lécué, F., Tucker, R., Bicer, V., Tommasi, P., Tallevi-Diotallevi, S., \& Sbodio, M. (2014). Predicting severity of road traffic congestion using semantic web technologies. In V. Presutti, C. d'Amato, F. Gandon, M. d'Aquin, S. Staab, \& A. Tordai (Eds.), The Semantic Web: Trends and Challenges (pp. 611-627). Cham: Springer International Publishing.

Lécué, F., \& Wu, J. (2017). Explaining and predicting abnormal expenses at large scale using knowledge graph based reasoning. Web Semantics, 44, 89-103.

Li, Z., Outbib, R., Giurgea, S., Hissel, D., Jemei, S., Giraud, A., \& Rosini, S. (2016). Online implementation of svm based fault diagnosis strategy for PEMFC systems. Applied energy, 164, 284-293. 
Lin, R.-H., Xi, X.-N., Wang, P.-N., Wu, B.-D., \& Tian, S.-M. (2019). Review on hydrogen fuel cell condition monitoring and prediction methods. International Journal of Hydrogen Energy, 44, 5488 - 5498. doi:https://doi.org/10.1016/j. ijhydene.2018.09.085. The 6th International Conference on Energy, Engineering and Environmental Engineering.

Liu, J., Li, Q., Chen, W., \& Cao, T. (2018). A discrete hidden markov model fault diagnosis strategy based on k-means clustering dedicated to pem fuel cell systems of tramways. International Journal of Hydrogen Energy, 43, 12428 - 12441.

Liu, J., Li, Q., Chen, W., Yan, Y., \& Wang, X. (2019). A fast fault diagnosis method of the pemfc system based on extreme learning machine and DempsterShafer evidence theory. IEEE Transactions on Transportation Electrification, 5, $271-284$.

Luta, D. N., \& Raji, A. K. (2019). Comparing fuzzy rule-based mppt techniques for fuel cell stack applications. Energy Procedia, 156, 177 - 182. 5th International Conference on Power and Energy Systems Engineering (CPESE 2018).

Ma, R., Yang, T., Breaz, E., Li, Z., Briois, P., \& Gao, F. (2018). Data-driven proton exchange membrane fuel cell degradation predication through deep learning method. Applied Energy, 231, $102-115$.

Mao, L., \& Jackson, L. (2016). Selection of optimal sensors for predicting performance of polymer electrolyte membrane fuel cell. Journal of Power Sources, 328, $151-160$.

Mao, L., Jackson, L., \& Dunnett, S. (2017). Fault diagnosis of practical polymer electrolyte membrane (PEM) fuel cell system with data-driven approaches. Fuel Cells, 17, 247-258.

Mao, L., Jackson, L. M., \& Davies, B. (2018). Effectiveness of a novel sensor selection algorithm in PEM fuel cell on-line diagnosis. IEEE Transactions on Industrial Electronics, 65, 7301-7310.

Nguyen, T. V., \& Knobbe, M. W. (2003). A liquid water management strategy for PEM fuel cell stacks. Journal of Power Sources, $114,70-79$.

Petrone, R., Zheng, Z., Hissel, D., Pra, M., Pianese, C., Sorrentino, M., Becherif, M., \& Yousfi-Steiner, N. (2013). A review on model-based diagnosis methodologies for PEMFCs. International Journal of Hydrogen Energy, 38 , 7077 7091. URL: http://www.sciencedirect.com/science/article/pii/S0360319913007465. doi:https://doi.org/10.1016/j. ijhydene.2013.03.106.

Pourkiaei, S. M., Ahmadi, M. H., \& Hasheminejad, S. M. (2016). Modeling and experimental verification of a 25w fabricated PEM fuel cell by parametric and gmdh-type neural network. Mechanics \& Industry, $17,105$.

Rahimzadeh, A., Ashtiani, F. Z., \& Okhovat, A. (2016). Application of adaptive neuro-fuzzy inference system as a reliable approach for prediction of oily wastewater microfiltration permeate volume. Journal of environmental chemical engineering, 4, 576-584.

Ramaswamy, N., Hakim, N., \& Mukerjee, S. (2008). Degradation mechanism study of perfluorinated proton exchange membrane under fuel cell operating conditions. Electrochimica Acta, 53, 3279-3295.

Ramos-Paja, C. A., Romero, A., Giral, R., Vidal-Idiarte, E., \& Martinez-Salamero, L. (2009). Fuzzy-based modelling technique for PEMFC electrical power generation systems emulation. IET Power Electronics, 2, 241-255. doi:10.1049/iet-pel.2008. 0008.

Roda, F., \& Musulin, E. (2014). An ontology-based framework to support intelligent data analysis of sensor measurements. Expert Systems with Applications, 41, 7914-7926.

Shams, M. B., Budman, H., \& Duever, T. (2011). Fault detection, identification and diagnosis using cusum based pca. Chemical Engineering Science, 66, 4488-4498.

Shao, M., Zhu, X.-J., Cao, H.-F., \& Shen, H.-F. (2014). An artificial neural network ensemble method for fault diagnosis of proton exchange membrane fuel cell system. Energy, 67, $268-275$. URL: http://www.sciencedirect.com/science/ article/pii/S0360544214001017. doi:https://doi.org/10.1016/j.energy.2014.01.079.

Silva, R., Gouriveau, R., Jemei, S., Hissel, D., Boulon, L., Agbossou, K., \& Steiner, N. Y. (2014). Proton exchange membrane fuel cell degradation prediction based on adaptive neuro-fuzzy inference systems. International Journal of Hydrogen Energy, $39,11128-11144$.

Sompalli, B., Litteer, B. A., Gu, W., \& Gasteiger, H. A. (2007). Membrane degradation at catalyst layer edges in PEMFC MEAs. Journal of The Electrochemical Society, 154, B1349-B1357.

Stoilos, G., \& Stamou, G. B. (2014). Hybrid query answering over DL ontologies. In Informal Proceedings of the 27th International Workshop on Description Logics (pp. 336-339).

Tsalapati, E., Jackson, T., Johnson, W., Jackson, L. M., Vasilyev, A., West, A. A., Mao, L., \& Davies, B. (2018). The role of semantic technologies in diagnostic and decision support for service systems. In Proceedings of the 51 st Hawaii International Conference on System Sciences, HICSS'18.

Vasilyev, A., Dunnett, S., \& Jackson, L. (2015). Model-based fault detection and isolation of PEM fuel cells using bond graphs. In Proceedings of European Safety and Reliability Conference, ESREL'15 (pp. 2481-2488).

Wang, J. (2017). System integration, durability and reliability of fuel cells: Challenges and solutions. Applied energy, 189, $460-479$.

Wasterlain, S., Candusso, D., Harel, F., Franois, X., \& Hissel, D. (2010). Diagnosis of a fuel cell stack using electrochemical impedance spectroscopy and bayesian networks. In 2010 IEEE Vehicle Power and Propulsion Conference (pp. 1-6). doi:10. 1109/VPPC. 2010.5729184.

Whiteley, M., Fly, A., Leigh, J., Dunnett, S., \& Jackson, L. (2015). Advanced reliability analysis of polymer electrolyte membrane fuel cells using petri-net analysis and fuel cell modelling techniques. International Journal of Hydrogen Energy, 40, 11550-11558.

Wieland, C., Schmid, O., Meiler, M., Wachtel, A., \& Linsler, D. (2009). Reliability computing of polymer-electrolyte-membrane fuel cell stacks through petri nets. Journal of Power Sources, 190, 34-39.

Yang, B., Lei, Y., Jia, F., \& Xing, S. (2019a). An intelligent fault diagnosis approach based on transfer learning from laboratory bearings to locomotive bearings. Mechanical Systems and Signal Processing, 122, $692-706$.

Yang, J., Xiong, W., Li, S., \& Xu, C. (2019b). Learning structured and non-redundant representations with deep neural 
networks. Pattern Recognition, 86, $224-235$.

Zhao, R., Yan, R., Chen, Z., Mao, K., Wang, P., \& Gao, R. (2019). Deep learning and its applications to machine health monitoring. Mechanical Systems and Signal Processing, 115, 213-237. doi:10.1016/j.ymssp.2018.05.050.

Zheng, Z., Petrone, R., Pra, M., Hissel, D., Becherif, M., Pianese, C., Steiner, N. Y., \& Sorrentino, M. (2013). A review on non-model based diagnosis methodologies for PEM fuel cell stacks and systems. International Journal of Hydrogen Energy, 38, 8914 - 8926. URL: http://www.sciencedirect.com/science/article/pii/S0360319913008550. doi:https://doi.org/ 10.1016/j.ijhydene.2013.04.007.

Zheng, Z., Pra, M.-C., Hissel, D., Becherif, M., Agbli, K.-S., \& Li, Y. (2014). A double-fuzzy diagnostic methodology dedicated to online fault diagnosis of proton exchange membrane fuel cell stacks. Journal of Power Sources, 271, 570 - 581. URL: http://www.sciencedirect.com/science/article/pii/S0378775314012117. doi:https://doi.org/10.1016/j. jpowsour.2014.07.157. 